\title{
A model of reporting and controlling outbreaks by public health agencies
}

\author{
Alexander E. Saak ${ }^{1}$ • David A. Hennessy ${ }^{2}$
}

Received: 23 December 2015 / Accepted: 20 April 2017 / Published online: 14 June 2017

(C) Springer-Verlag Berlin Heidelberg 2017

\begin{abstract}
We study a model of detection and control of an infectious disease by local health agencies in the presence of imperfect information about the likelihood of an outbreak and limited diagnostic capacity that is insufficient to determine the prevalence of disease in all localities in a timely manner. When shared diagnostic capacity is rationed based on reports of symptoms, the decision to report symptoms or not creates a trade-off. On the one hand, rigorous testing of one's locality allows the health agency in that locality to make an informed disease control decision. On the other hand, reducing the availability of diagnostic capacity for the other localities increases the probability that the disease will spread from an untested area where the disease is not controlled in the absence of more evidence of an outbreak. Symptoms are over-reported (respectively, reported truthfully, or under-reported) when the cost of disease control is sufficiently small (respectively, in some intermediate range, or sufficiently large). The rates of reporting suspicions decrease as the cost of disease control increases because the localities remain exposed more often and the health agencies prefer to reduce the risk of disease transmission by letting other agencies become informed. If the disease incidence decreases or infectiousness increases, symptoms are reported less frequently. If the precision of private signals increases, the extent of over-reporting of symptoms may increase. For different values of the parameters it can be socially
\end{abstract}

$\triangle \quad$ Alexander E. Saak

asaak@cgiar.org

David A. Hennessy

hennes64@anr.msu.edu

1 Markets, Trade, and Institutions Division, International Food Policy Research Institute, $2033 \mathrm{~K}$

ST NW, Washington, DC 20006, USA

2 Department of Agricultural, Food, and Resource Economics, Michigan State University, East Lansing, MI 48824, USA 
optimal to subsidize or tax requests for additional investigations and confirmatory testing.

Keywords Cheap talk - Infectious disease - Private information · Public health agency · Testing

JEL Classification D23 - D62 - D73 - D83 - L23

\section{Introduction}

This paper is concerned with the incentives of public health agencies to truthfully report and efficiently control outbreaks of communicable diseases. When an outbreak of an infectious disease is suspected, a local health agency may notify a state or federal agency and request additional resources to investigate and, if necessary, contain it. However, due to capacity constraints, state and federal health agencies may not be able to grant all such requests which may give an incentive to local agencies to request help strategically.

Disease outbreaks are episodic by nature. Spasmodically intense demand for services do not create market failures, per se. However when the services in question are intended to inform on externalities to others, then the need to ration can add to the externality management problem. Public health services are acutely aware of the technical problems that arise from rationing for the diagnostic laboratories and other resources allocated to investigate possible disease outbreaks (Metzler et al. 2010; Gates 2015; Schäferhoff et al. 2015). Technology transfer, standards setting, training subventions and efficient resource sharing have been parts of public policy for many years. In the USA, the Centers for Disease Control and Prevention (CDC) has facilitated a partnership of shared federal, state and international diagnostic human health laboratories, the Laboratory Response Network, since 1999. A similar entity, the Veterinary Laboratory Investigation and Response Network, is coordinated by the US Food and Drug Administration. The World Health Organization has a Global Outbreak Alert and Response Network in place and is responsible for strengthening capacity in certain countries, though the 2015 Ebola outbreak and other recent events have revealed a consensus view that these resources are far from adequate (Gates 2015). Many countries also have contingent contracts and other plans in place to have available appropriately trained personnel in the event of a demand surge.

Our concern is not with the level of resources available, but with how these limited resources are used. This paper's contributions to the literature are to identify and study strategic motives for reporting communicable diseases in the presence of rationed tests, and to explain implications for disease prevention and control. ${ }^{1}$ Our case is that in which a small number (two) of local health agencies (henceforth referred to as agents) in adjacent localities compete for the limited detection/diagnostic capacity (an investigation team or laboratory space). The disease spreads in two stages. In the

\footnotetext{
1 The model studied in the paper can also be applied to other contexts such as controlling bank runs and financial contagion (Brown et al. 2016).
} 
first stage, initial outbreaks may or may not occur in each locality. In the second stage, the disease (if any) spreads within and across localities unless measures are taken to contain the outbreak. Each agent privately observes an imperfect signal of the initial disease prevalence in her locality. For example, symptoms of an outbreak may include an increase in the number or severity of cases of human or animal illness, or results of preliminary, inconclusive food or water tests for pathogen contamination. Then the agents can use this information to request a confirmatory, conclusive investigation and testing, but the request may not be granted if demand is high for the services of the shared investigative unit (at most one of the agents can be tested). The initial information in addition to information arising from their investigation request's outcome (that is, the allocation of the test) and the results of an investigation itself (if any) can be used to infer the overall disease prevalence and the likelihood of subsequent spread across localities.

Upon learning and inferring from all available information, the agent can take a costly action such as implementing a vaccination program, quarantine of susceptible population, restrictions on travel or animal movement, cleaning and disinfecting contaminated areas, or hiring additional medical personnel. For that agent, taking this action would cure (that is, the locality would become disease-free) a disease already present in the agent's locality and would protect against contracting a disease not initially present in the locality. Furthermore, in taking the action the agent would generate a positive externality because this agent (the agent's locality) would pose lower risk for others. ${ }^{2}$ We are interested in understanding the agents' joint decisions to request an investigation and exert disease control efforts. We show that even though in equilibrium the agents take too few precautions, they can either under-request or over-request additional investigations/testing, thus creating inefficiencies in the choice of public health system efforts to both assess and control the disease. An important finding is that, all else equal, the agents report suspicions less often if the cost of disease control increases. This happens because the agents respond to a greater cost of disease control by controlling the disease less often which in turn increases the risk of disease transmission from an agent who is uncertain about the disease prevalence in her locality. To reduce this risk of disease transmission, each agent is more willing to give up the testing capacity as doing so increases the probability that the other agent will exert the appropriate disease control effort.

Our analysis of this sequential game is based on the solution concept of perfect Bayesian equilibrium and proceeds by first solving for the information-conditioned beliefs and disease control choices (in Sect. 3) and then using them to infer demands for confirmatory testing (Proposition 1). Confirmatory testing has two effects on overall disease control efforts. On the one hand, better informed agents are more likely to control the disease if necessary (the information effect). On the other hand, testing

\footnotetext{
2 In their Table 2, Funk et al. (2010) seek to place behavioral change within the context of the standard Kermack and McKendrick susceptible-infected-recovered model so that behavior can change an agent's disease state or change model parameters or contact structure. That model provides limited openings for studying information externalities, and so our model does not fall naturally within their Table 2 framework. Nor do we formally address contact structure. If one were to place our setting within that framework, it would involve changing both an agent's disease state and the model disease parameter that maps contact into infection.
} 
decreases the equilibrium disease control efforts by those who remain untested (the externality effect). While the information effect of testing is completely determined by the initial private information about the disease incidence, the externality effect is greater when demand for testing increases because agents, who are crowded out of testing, exercise less care in controlling the disease.

Both the information and externality effects are taken into account in the first stage of the game when demand for external testing is determined. Although in equilibrium the reports of suspicions are positively correlated with symptoms, symptoms can be over-reported or under-reported depending on the relative strengths of the information and externality effects, that is, concerns with (a) learning about the health status in one's locality in order to apply treatment if initially infected and avoid unnecessary expenditures if initially disease-free and (b) the disease transmission risk from the locality that requested a test but could not be tested due to the capacity constraint. However, a simple tax or subsidy for getting tested (such a scheme can take the form of state/federal assistance to local health agencies or fees for the use of state/federal investigative unit) can provide the incentives to report symptoms/suspicions truthfully and maximize the social benefits of rigorous testing as long as the private and social benefits of testing are not too misaligned.

\subsection{Literature review}

Classical analysis of epidemic outbreaks has largely modeled dynamical systems of populations, see Daley and Gani (1999). These systems have provided limited roles for behavioral responses on the part of the public health system, firms, and the subject population. Much is missing, as indicated by recent scrutiny of incentives for supply and demand in vaccine markets. Bauch et al. (2003) and others have sought to better understand stubborn resistance to public vaccination programs. They have focused on a divergence between small private incentives to vaccinate in the presence of a small cost when many others have already vaccinated and the potentially much larger public incentives to ensure herd immunity from vaccination. Ward (2014) shows empirically that certain vulnerable sub-populations benefit from high influenza vaccination rates in the community at large.

Given the potential impact of disease outbreak and spread, it is not surprising that the economics of managing the spread of an infectious disease has also received attention. An example is Klepac et al. (2011), who study prevention, vaccination and treatment choices during the onset or course of an epidemic. Another is Klepac et al. (2012), who consider the optimal mix of vaccination and palliative care taking into account the budget constraint. Nguyen and Carlson (2016) also use a SIR (susceptibleinfected-recovered) model to simulate the allocation of scarce vaccine in the event of an epidemic in the presence of time delays and interacting populations. ${ }^{3}$ Our work is distinct in that it studies communication and learning about the extent of a potential

\footnotetext{
3 Also related is Klepac et al. (2016), who use a game-theoretic framework to explore when international agreements can help countries implement efficient vaccination strategies. Goenka and Liu (2012) and Goenka et al. (2014) study the impact of infectious disease on the economy and consider the role of isolation and vaccination in controlling diseases in a dynamic equilibrium framework.
} 
outbreak before any efforts to control the disease are taken. Our paper presents novel results about the inefficiencies that may arise in disease management in adjacent areas when there is uncertainty about the initial disease prevalence and limited capacity to test for the presence of an infection. To carry out the analysis, our model considers just two times points (at the first time point, infection can be introduced from outside of the region, and at the second time point, the infection can spread across the region from one area to another unless the appropriate disease control measures are taken). Although, compared to the analysis based on the spatial /transboundary SIR models, our model says relatively little about how the disease prevalence evolves over time, the analysis sheds novel light on the potential problems in early detection.

A sub-discipline at the interface between epidemiology and the behavioral sciences has emerged to address the bounds that human actions have placed on epidemiological tools for policy analysis and for supporting field work in particular disease management campaigns, see Funk et al. (2010). Perhaps the most prominent approach to integrating behavior into such models has been the endogenization of social distancing, namely allowing agents to adapt social interaction behavior to the presence and extent of a disease and also allowing policy makers to seek to engineer such adaptation, as in Fenichel et al. (2011) and Perrings et al. (2014). Funk et al. (2015) outline a variety of other behavioral responses that warrant attention. One of these, outlined in their Challenge 4, asks for more research on how diagnosis and reporting behavior change during the course of a disease event. ${ }^{4}$ Such voluntary action and information issues comprise the essence of our concern, but where we also emphasize scarcity of resources available to address the disease.

The role of test choice, absent a capacity constraint, has arisen elsewhere in the literature. For example, Shavell (1994) has considered when producers choose to undergo a third party test on product quality and then on whether to make test results publicly available. One finding is that requiring all who test and find a product defect to make their test results public may undermine demand for testing. Philipson and Posner (1995) have made this point in the context of testing for infectious diseases. Kremer (1996) shows conceptually that responses to infectious disease test information are likely to be heterogeneous in regard to preventive behavior, and may be a wash upon aggregation while Boozer and Philipson (2000) hold that responses will be largest among those who are most surprised by the results. As far as we are aware, no work has considered strategic dimensions to test availability and demand for testing which

\footnotetext{
4 There is recent work that considers the effects of private information about the prevalence of infectious disease on how the disease spreads. Malani and Laxminarayan (2011) study incentives for a country to report imperfect information about a disease outbreak to its trading partner, resulting in a trade-off between medical assistance and trade sanctions. Mesnard and Seabright (2009) investigate the effects of restricting migration in a setting with two population centers and with private information about health. Adhvaryu (2015) and Cohen et al. (2015) study the effects of diagnostic tests on the treatment of malaria in the presence of learning about therapeutic effectiveness and subsidies for treatment and diagnostics but do not explicitly take into account the effects of the spread of disease.
} 
may signal to others that the disease prevalence is greater than expected as well as that care will be taken to control the disease. ${ }^{5}$

Our paper also contributes to a large literature on cheap talk with multiple senders that builds on Crawford and Sobel (1982). The models in Gilligan and Krehbiel (1989), Epstein (1998), and Krishna and Morgan (2001) make different assumptions about the configurations of the senders' preferences relative to one another and the receiver (decision-maker), but the state space is one-dimensional and senders have perfect information about the realized state. Similar to our case, in the settings in Wolinsky (2002) and McGee and Yang (2013) experts have partial, non-overlapping, and complementary information. These papers show that partial communication among experts may elicit the greatest amount of information, and that the senders' information transmissions exhibit strategic complementarity. Here we allow for imperfect signals about the states of the world and for two-way communication whereas our agents report and receive information about disease incidence. The problem considered here is not just one of cheap talk as the report made by an agent (location) gets a response from the central testing agency which rations testing and influences the agents' subsequent choices.

In models of multi-dimensional cheap talk with two perfectly informed senders and a receiver who makes two decisions, Battaglini (2002) and Ambrus and Takahashi (2008) show that equilibrium communication can fully reveal private information. In contrast, we find that communication may or may not be truthful when senders have non-overlapping, imperfect information. In Austen-Smith (1993) and Morgan and Stocken (2008) experts observe conditionally independent signals regarding the state of the world, which gives rise to an information congestion effect whereas transmission of information by one agent reduces the sensitivity of the receiver's decision to the report of another agent, and thus reduces the incentives to transmit information. In our setting, agents strategically manipulate not only the policy outcome (test allocation) but also each other's actions (disease control efforts).

The rest of the paper is organized as follows. In Sect. 2, we present a simple twostage model of spread of a controllable infectious disease between two localities in the presence of imperfect information about the disease incidence and limited diagnostic testing. In Sects. 3 and 4, we characterize equilibrium disease control efforts and demand for testing. In Sect. 5, we consider the optimal subsidy/tax scheme. Section 6 extends the model to accommodate more than two agents/localities. Section 7 concludes.

\footnotetext{
5 Motivated by the potential for subsidies in less developed countries, a recent line of literature has considered how test information on HIV/AIDS might affect behavior. For a randomized treatment trial in Malawi, Thornton (2008) finds that information did impact later condom purchase choices but the impacts were not large. Also by way of a randomized trial on HIV/AIDS testing in sub-Saharan Africa, Gong (2014) finds that test positive (resp., negative) individuals subsequently increase (decrease) risky behavior. Godlonton and Thornton (2012) show that own demand for HIV/AIDS testing increases with neighbors' decisions to do so. Their 2013 work goes further to ask how others' test information affects own behavior. They find that individuals who learn that disease prevalence is less than expected in a region are less cautious in sexual activity.
} 


\section{Model}

\subsection{Set-up}

There are two identical agents/localities, $A$ and $B$, and two time periods, 0 and $1 .{ }^{6}$ In each period, each agent is in one of two disease states: infected, $S=1$, or uninfected, $H=0$. Let $\theta_{i, t} \in\{S, H\}$ denote the agent $i$ 's disease state in period $t$. In period 1 , each agent can put in effort to control the disease or not, $e_{i} \in\{0,1\}$, at cost $c e_{i}$, where $0<c<1$. The loss to agent $i$ from the disease expressed in monetary units is given by $\theta_{i, 1}$.

\subsection{Disease epidemiology}

In period 0 , each agent is infected with probability (the base rate) $\alpha$, that is,

$$
\operatorname{Pr}\left(\theta_{i, 0}=S\right)=1-\operatorname{Pr}\left(\theta_{i, 0}=H\right)=\alpha .
$$

We will refer to $\alpha$ as the initial incidence of the disease. The initial disease states, $\theta_{A, 0}$ or $\theta_{B, 0}$, are drawn independently. In period 1 , after the agents choose whether to control disease, the disease stochastically spreads as follows

$$
\operatorname{Pr}\left(\theta_{i, 1}=S \mid \theta_{A, 0}, \theta_{B, 0}, e_{A}, e_{B}\right)=\left[\theta_{i, 0}+\delta\left(1-\theta_{i, 0}\right)\left(1-e_{-i}\right) \theta_{-i, 0}\right]\left(1-e_{i}\right),
$$

where $\delta \in(0,1]$ is the infectiousness of the disease, $-i=B$ if $i=A$ and $-i=A$ if $i=B$. An agent (say agent $A$ ) is infected in period 1 if she does not control the disease, $e_{A}=0$, and becomes infected either in period $0,\left(\theta_{A, 0}, \theta_{A, 1}\right)=(S, S)$, or in period $1,\left(\theta_{A, 0}, \theta_{A, 1}\right)=(H, S)$. The latter event happens if the other agent (agent $\left.B\right)$ is infected in period $0, \theta_{B, 0}=S$, does not control the disease, $e_{B}=0$, and the disease spreads from one agent to another (with probability $\delta$ ).

It should be noted that allowing for uncertainty about the virulence of the disease $(\delta)$ leads to an equivalent analysis as long as $\delta$ is common to both agents and drawn independently from the initial disease states (this is formally shown in Appendix D). Also, note that we assume that treatment implies recuperation in the end of the second period as an agent who controls the disease, $e_{i}=1$, is always uninfected in period 1 (for any realizations of the initial disease states and the effort of the other agent). Finally, it is worth observing that the risk of disease transmission is endogenous in our model because it decreases with the disease control effort chosen by the infected agent. $^{7}$

\footnotetext{
6 We will use the words "agent" and "locality" interchangeably, where "agent" could be thought of as a local public health agency and "locality" is the geographic area under the authority of that agency.

7 Because the expected loss from infection is decreasing in the agent's own disease control effort and the disease control effort chosen by the other agent, the disease control choices are strategic substitutes.
} 


\subsection{Information about disease incidence in period 0}

The initial disease states are unknown but each agent $i$ privately observes a signal, $Z_{i}$, that is correlated with her actual initial disease state as follows

$$
\begin{aligned}
\operatorname{Pr}\left(Z_{i}=s \mid \theta_{i, 0}=S\right) & =1-\operatorname{Pr}\left(Z_{i}=h \mid \theta_{i, 0}=S\right)=\beta, \\
\operatorname{Pr}\left(Z_{i}=s \mid \theta_{i, 0}=H\right) & =1-\operatorname{Pr}\left(Z_{i}=h \mid \theta_{i, 0}=H\right)=1-\beta
\end{aligned}
$$

where $\beta \in\left(\frac{1}{2}, 1\right)$ is the precision of signal $Z_{i}$. We will refer to an agent with signal $Z_{i}=s$ as "symptomatic", and to an agent with signal $Z_{i}=h$ as "asymptomatic", and we let $z$ denote a typical realization of $Z_{i}$. Let

$$
\begin{aligned}
& \lambda_{S \mid s} \equiv \operatorname{Pr}\left(\theta_{i, 0}=S \mid Z_{i}=s\right)=\frac{\alpha \beta}{\alpha \beta+(1-\alpha)(1-\beta)} \text { and } \\
& \lambda_{S \mid h} \equiv \operatorname{Pr}\left(\theta_{i, 0}=S \mid Z_{i}=h\right)=\frac{\alpha(1-\beta)}{\alpha(1-\beta)+(1-\alpha) \beta}
\end{aligned}
$$

denote the probabilities of being initially infected conditional on signal $z \in\{s, h\}$, where $\lambda_{S \mid h}<\alpha<\lambda_{S \mid s}$, and let $g \equiv \operatorname{Pr}\left(Z_{i}=s\right)=\alpha \beta+(1-\alpha)(1-\beta)$ denote the unconditional probability with which an agent observes symptoms.

\subsection{Reports and testing}

There is a diagnostic capacity to test the initial disease state of agent $A$ or $B, \theta_{A, 0}$ or $\theta_{B, 0}$, but not both. The test reveals the true state with probability 1 . The assumption that the test is perfectly informative simplifies the analysis but is not essential to our results (see Sect. 4.1). Let $T \in\{A, B, \varnothing\}$ denote the identity of the tested agent, where $T=\varnothing$ means that neither are tested. The test allocation procedure is as follows: First, each agent $i$ reports suspicions of disease, $r_{i}=s$, or not, $r_{i}=h$. Second, the test is allocated in accordance with the following rule:

$$
\begin{aligned}
& \operatorname{Pr}\left(T=A \mid r_{A}, r_{B}\right)= \begin{cases}1, & \text { if }\left(r_{A}, r_{B}\right)=(s, h) \\
\frac{1}{2}, & \text { if }\left(r_{A}, r_{B}\right)=(s, s) \\
0, & \text { if }\left(r_{A}, r_{B}\right) \in\{(h, s),(h, h)\}\end{cases} \\
& \operatorname{Pr}\left(T=B \mid r_{A}, r_{B}\right)=\operatorname{Pr}\left(T=A \mid r_{B}, r_{A}\right), \\
& \operatorname{Pr}\left(T=\varnothing \mid r_{A}, r_{B}\right)=\left\{\begin{array}{ll}
0, & \text { if }\left(r_{A}, r_{B}\right) \in\{(s, s),(h, s),(s, h)\} \\
1, & \text { if }\left(r_{A}, r_{B}\right)=(h, h)
\end{array} .\right.
\end{aligned}
$$

For concreteness, we assume that the reports, the test allocation, and the test outcome are all private information, and that reporting a suspicion is costless. ${ }^{8}$ We consider what happens when the testing agency charges a fee for testing in Sect. 5.

\footnotetext{
8 The analysis is qualitatively similar if the reports and/or test outcomes are public information (see Sect. 4). In reality, the national authority can disclose the reports of suspicions and test outcomes to local health authorities. The simplifying assumption that public health authorities learn about the risks of disease transmission
} 

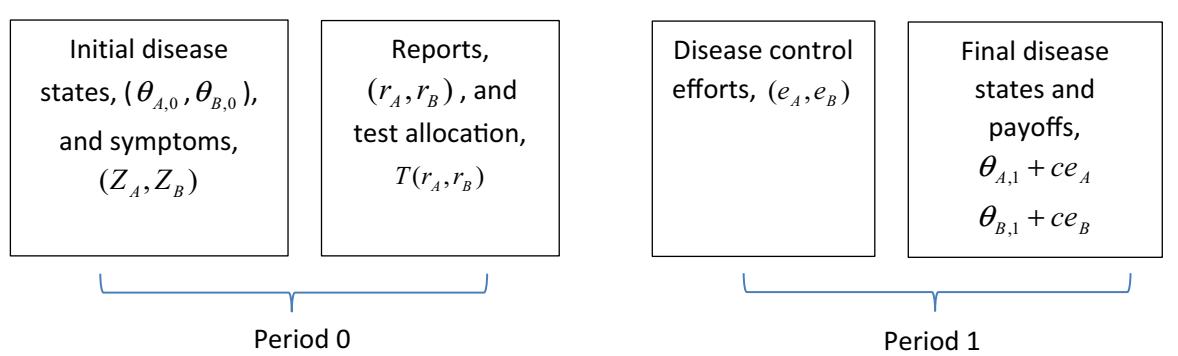

Fig. 1 Timing of events

\subsection{Timing of events}

The timing of events is as follows (Fig. 1):

1. Initial disease states $\theta_{A, 0}$ and $\theta_{B, 0}$ are realized.

2. Each agent privately observes signal $Z_{i}$.

3. Each agent privately reports suspicions or not, $r_{i}$.

4. Each player privately observes either the test outcome, $\theta_{i, 0}$, if $T=i$, or event $\{i \notin T\}$.

5. Agents simultaneously choose to control disease or not, $e_{i}$.

6. Final disease states and the payoffs are realized, $\theta_{i, 1}+c e_{i}$.

\subsection{Solution concept}

Let $y_{i}:\{s, h\} \rightarrow[0,1]$ and $x_{i}:\{s, h\} \times\{s, h\} \times\{S, H, \varnothing\} \rightarrow[0,1]$ denote agent $i$ 's mixed reporting and effort strategies, where the first component in the cross product is the set of possible realizations of private signals, the second component is the set of possible reports, and the third is the set of possible testing outcomes where the agent either learns her initial disease state or observes that she is not selected for testing (untested). Also, let $\sigma_{i}:\{s, h\} \times\{S, H, \varnothing\} \rightarrow[0,1]$ denote the agent's belief about the other agent's signal as a function of the agent's report and test outcome. We solve for a Perfect Bayesian Equilibrium, which requires that:

(1) Given agent $j$ 's reporting and effort strategies, $y_{j}$ and $x_{j}$, agent $i$ 's reporting and effort strategies, $y_{i}$ and $x_{i}$, induce a Nash equilibrium in every subgame (in period 0 and period 1 ),

(2) agent $i$ 's belief about agent $j$ 's signal is derived from agent $j$ 's reporting strategy and agent $i$ 's information in the beginning of period 1 according to Bayes rule whenever possible.

We consider a symmetric equilibrium where both agents play the same strategies and drop the subscripts $i$ from the strategies and beliefs. Let $x_{\theta}$ denote the probability with which an agent for whom a test reveals her initial disease state $\theta \in\{S, H\}$, controls disease, 


$$
x_{\theta}=\operatorname{Pr}\left(e_{i}=1 \mid \theta_{i, 0}=\theta\right) \in[0,1] .
$$

Let $x_{z u}$ denote the probability with which an untested agent with signal $z \in\{s, h\}$ who reported suspicions, controls disease,

$$
x_{z u}=\operatorname{Pr}\left(e_{i}=1 \mid Z_{i}=z, r_{i}=s, T \neq i\right) \in[0,1] .
$$

Let $x_{z h}$ denote the probability with which an (necessarily untested) agent with signal $z \in\{s, h\}$ who did not report suspicions, controls disease,

$$
x_{z h}=\operatorname{Pr}\left(e_{i}=1 \mid Z_{i}=z, r_{i}=h\right) \in[0,1] .
$$

Let $y_{z}$ denote the probability with which an agent with signal $z \in\{s, h\}$ reports suspicions,

$$
y_{z}=\operatorname{Pr}\left(r_{i}=s \mid Z_{i}=z\right) \in[0,1]
$$

Also, let $\sigma_{h}\left(\sigma_{s u}\right.$ and $\sigma_{\theta}$, respectively) denote the posterior belief of an agent that the other agent observed symptoms after reporting $h$ (respectively, reporting $s$ and not being selected for testing, and reporting $s$ and learning one's actual initial disease state).

Applying backward induction, we will first find the equilibrium beliefs, $\sigma_{h}^{*}, \sigma_{s u}^{*}, \sigma_{S}^{*}, \sigma_{H}^{*}$, and disease control strategies that the agents adopt in period 1 after the test has been allocated, $x_{S}^{*}, x_{H}^{*}, x_{s u}^{*}, x_{s h}^{*}, x_{h u}^{*}, x_{h h}^{*}$, for given probabilities with which the agents report suspicions in period 0 (in Sect. 3). Second, we will find the equilibrium probabilities with which agents report suspicions in period $0, y_{s}^{*}, y_{h}^{*}$, given the equilibrium beliefs about one another's private information and disease control strategies adopted in period 1 (in Sect. 4).

Note that the agents are interconnected because (a) the diagnostic capacity is limited, and (b) disease can spread from one agent to another. When deciding whether or not to report suspicions the forward-looking agents take into account that the disease control efforts and reporting strategies depend on the information about the initial disease incidence available to each agent. Because the risk of disease transmission is endogenous, the agents may have an incentive to manipulate each other's information through the test allocation.

\section{Disease control rates}

We begin by determining the optimal disease control strategies after the test has been allocated.

\subsection{Agent who tested positive}

A tested agent who learns that she is initially infected, $\theta_{i, 0}=S(=1)$ achieves the smallest loss if she controls the disease since the cost of disease control is smaller than the damage from the disease: 


$$
x_{S}^{*}=1=\underset{x}{\arg \min 1-x+x c} .
$$

Note that this implies that in equilibrium a tested agent is not infectious. Thus, agents may prefer not to demand to be tested as long as doing so increases the probability that the other agent is tested. In this case, the agent's posterior belief about the type (that is, the private signal) of the other agent is given by

$$
\sigma_{S}^{*}=\frac{g\left(1-y_{s}\right)+\frac{1}{2} g y_{s}}{g\left(1-y_{s}\right)+\frac{1}{2} g y_{s}+(1-g)\left(1-y_{h}\right)+\frac{1}{2}(1-g) y_{h}},
$$

and has no effect on the disease control effort in (1). To understand how the belief is calculated, note that the selection of a given agent who reported suspicions is guaranteed if the other agent does not report suspicions, which happens with probability $g\left(1-y_{s}\right)+(1-g)\left(1-y_{h}\right)$, or her selection occurs by random assignment if the other agent also reports suspicions, which happens with probability, $\frac{1}{2}\left[g y_{s}+(1-g) y_{h}\right]$.

\subsection{Agent who reported suspicions but is untested}

An untested agent who reported suspicions can infer that the other agent was tested. As shown above, this implies that this agent faces no disease transmission risk. Therefore, if an untested agent with signal $Z_{i}=z$, who reported suspicions, does not put in disease control effort she becomes infected with probability $\lambda_{S \mid z}$, that is,

$$
E\left[\theta_{i, 1} \mid Z_{i}=z, T=j, e_{i}=0\right]=E\left[\theta_{i, 0} \mid Z_{i}=z\right]=\lambda_{S \mid z},
$$

where $j=A$ if $i=B$, and $j=B$ if $i=A$. Hence, the equilibrium disease control probability of an untested agent who reported suspicions is given by

$$
x_{z u}^{*}=1_{\lambda_{S \mid z} \geq c}=\underset{x}{\arg \min }(1-x) \lambda_{S \mid z}+x c .
$$

Again, her belief about the other agent's type,

$$
\sigma_{s u}^{*}=\frac{\frac{1}{2} g y_{s}}{\frac{1}{2} g y_{s}+\frac{1}{2}(1-g) y_{h}},
$$

has no effect on her disease control effort.

The two threshold values of the cost of disease control, that is, the conditional initial disease incidence, $\lambda_{S \mid h}$ and $\lambda_{S \mid s}$, depend on the unconditional initial disease incidence, $\alpha$, and the precision of private signals, $\beta$. As the unconditional disease incidence increases, $\lambda_{S \mid h}$ and $\lambda_{S \mid s}$ both increase, and the disease control efforts of untested reporters of suspicions weakly increase as well. Furthermore, it can be verified that $\partial\left(\lambda_{S \mid s}-\lambda_{S \mid h}\right) / \partial \alpha \geq(<) 0$ as $\alpha \leq(>) \frac{1}{2}$, that is, an increase in the disease incidence has a relatively greater effect on the beliefs of a symptomatic or an asymptomatic agent depending on the current disease incidence. As a result, as $\alpha$ increases, the interval 
of the disease control costs for which only an asymptomatic untested reporter of suspicions does not put in the disease control effort, $\left[\lambda_{S \mid h}(\alpha, \beta), \lambda_{S \mid s}(\alpha, \beta)\right]$, expands as long the disease remains unlikely, $\alpha<\frac{1}{2}$, and then contracts once the disease becomes likely, $\alpha>\frac{1}{2}$.

In contrast, an increase in $\beta$ has the opposite effects on $\lambda_{S \mid h}$ and $\lambda_{S \mid s}$. While a symptomatic agent becomes more certain that she has already been infected, an asymptomatic agent becomes more certain that she has not yet been infected. Therefore, the interval of the disease control costs for which only an asymptomatic untested reporter of suspicions is potentially infectious, $\left[\lambda_{S \mid h}(\alpha, \beta), \lambda_{S \mid s}(\alpha, \beta)\right]$, expands whenever private signals of the initial disease state become more precise.

It is also worth pointing out that whether an untested symptomatic agent who reported suspicions is more infectious than an untested asymptomatic agent who reported suspicions depends on the cost of disease control. If $c \leq \lambda_{S \mid h}$, no untested agent who reported suspicions is infectious because an untested agent of any type prefers to control the disease. If $\lambda_{S \mid h}<c \leq \lambda_{S \mid s}$, then only an asymptomatic untested reporter of suspicions is potentially infectious. Finally, if $c>\lambda_{S \mid s}$, then all untested reporters of suspicions are potentially infectious, and symptomatic untested reporters are more so, since their disease control rates drop to zero.

\subsection{Agent who did not report suspicions}

The agent who did not report suspicions never learns whether the other agent was selected for testing because, with probability one, she will not be selected for testing. Therefore, a non-reporter $i$ assigns probability $\sigma_{h}^{*}=g$ that the other agent observed signal $Z_{j}=s$ and the complimentary probability $1-g$ to signal $Z_{j}=h$, as the non-reporter receives no new information to update her beliefs about the other agent, $j \neq i$. Hence, from the point of view of the non-reporter $i$, the other agent with signal $Z_{j}=z \in\{s, h\}$ is infectious if she (1) is infected in period 0 (with probability $\lambda_{S \mid z}$ ), and (2) does not report suspicions (with probability $1-y_{z}$ ), and (3) does not control the disease (with probability $1-x_{z h}^{*}$ ). Aggregation over signals yields

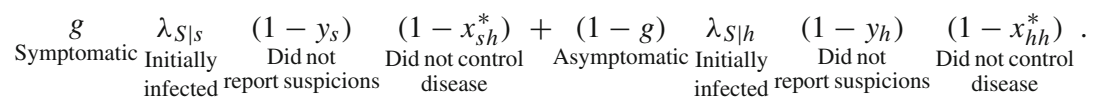

Note that the probability of becoming infected in period 1 decreases with an increase in the disease control efforts exerted by non-reporters of suspicions, $x_{s h}^{*}$ and $x_{h h}^{*}$, as well as with the probabilities of reporting suspicions, $y_{s}$ and $y_{h}$, because a tested agent is not infectious.

Using (3), the optimal disease control effort of an agent who did not report suspicions (and who expects that the other agent, if she does not report suspicions, adopts disease control strategies $x_{s h}^{*}, x_{h h}^{*}$ ) is given by

$$
\begin{aligned}
& x_{z h}^{*} \in \underset{x}{\arg \min }(1-x)\left\{\lambda_{S \mid z}+\left(1-\lambda_{S \mid z}\right) \delta\left[g\left(1-y_{s}\right) \lambda_{S \mid s}\left(1-x_{s h}^{*}\right)\right.\right. \\
& \left.\left.\quad+(1-g)\left(1-y_{h}\right) \lambda_{S \mid h}\left(1-x_{h h}^{*}\right)\right]\right\}+x c
\end{aligned}
$$


for $z \in\{s, h\}$. To understand (4), note that a non-reporter $i$ with signal $Z_{i}=z$ becomes infected in period 1 either because she was infected in period 0 , which happens with probability $\lambda_{S \mid z}$, or because the disease spreads from infectious agent $j$ in period 1. The latter event happens with the probability calculated in (3). It bears explicit mention that the objective function in (4) presumes that local public health officials place no weight on how its actions affect others. This may be because locally elected officials provide the incentives that local public health officials respond to. Or it could be because, while central administration provides incentives, these incentives reward based on management and disease costs incurred within the locality.

Note that the only unknown disease control efforts in problem (4) are those of a nonreporter. The reason is that the other agent is either another non-reporter, or a tested reporter who, as shown previously, is not infectious in equilibrium. Therefore, solving jointly the optimality conditions for the problems (4), we can find the equilibrium probabilities of exerting disease control efforts by both types of agents who do not report suspicions, ${ }^{9}$

$$
\begin{gathered}
x_{s h}^{*}\left(y_{s}, y_{h}\right)=\left\{\begin{array}{l}
0, \text { if } \lambda_{S \mid s}+\left(1-\lambda_{S \mid s}\right) \delta\left(g\left(1-y_{s}\right) \lambda_{S \mid s}+(1-g)\left(1-y_{h}\right) \lambda_{S \mid h}\right)<c \\
1-\frac{c-\lambda_{S \mid s}-\left(1-\lambda_{S \mid s}\right) \delta(1-g)\left(1-y_{h}\right) \lambda_{S \mid h}}{\left(1-\lambda_{S \mid s}\right) \delta g\left(1-y_{s}\right) \lambda_{S \mid s}}, \text { if } \lambda_{S \mid s}+\left(1-\lambda_{S \mid s}\right) \delta(1-g)\left(1-y_{h}\right) \lambda_{S \mid h} \\
\leq c \leq \lambda_{S \mid s}+\left(1-\lambda_{S \mid s}\right) \delta\left(g\left(1-y_{s}\right) \lambda_{S \mid s}+(1-g)\left(1-y_{h}\right) \lambda_{S \mid h}\right) \\
1, \text { if } c<\lambda_{S \mid s}+\left(1-\lambda_{S \mid s}\right) \delta(1-g)\left(1-y_{h}\right) \lambda_{S \mid h}
\end{array}\right. \\
x_{h h}^{*}\left(y_{s}, y_{h}\right)=\left\{\begin{array}{c}
0, \text { if } \lambda_{S \mid h}+\left(1-\lambda_{S \mid h}\right) \delta(1-g)\left(1-y_{h}\right) \lambda_{S \mid h}<c \\
1-\frac{c-\lambda_{S \mid h}}{\left(1-\lambda_{S \mid h}\right) \delta(1-g)\left(1-y_{h}\right) \lambda_{S \mid h},}, \text { if } \lambda_{S \mid h} \leq c \leq \lambda_{S \mid h} \\
+\left(1-\lambda_{S \mid h}\right) \delta(1-g)\left(1-y_{h}\right) \lambda_{S \mid h} \\
1, \text { if } c<\lambda_{S \mid h}
\end{array}\right.
\end{gathered}
$$

If the cost of disease control is sufficiently great, even a symptomatic non-reporter does not control the disease, although the other non-reporter is potentially infectious. For $\lambda_{S \mid s}+\left(1-\lambda_{S \mid s}\right) \delta(1-g)\left(1-y_{h}\right) \lambda_{S \mid h} \leq c \leq \lambda_{S \mid s}+\left(1-\lambda_{S \mid s}\right) \delta\left(g\left(1-y_{s}\right) \lambda_{S \mid s}+\right.$ $\left.(1-g)\left(1-y_{h}\right) \lambda_{S \mid h}\right)$ and $\lambda_{S \mid h} \leq c \leq \lambda_{S \mid h}+\left(1-\lambda_{S \mid h}\right) \delta(1-g)\left(1-y_{h}\right) \lambda_{S \mid h}$, the equilibrium disease control rates are obtained by solving the indifference condition for problem (4),

$$
\lambda_{S \mid z}+\left(1-\lambda_{S \mid z}\right) \delta\left(g\left(1-y_{S}\right) \lambda_{S \mid s}\left(1-x_{s h}^{*}\right)+(1-g)\left(1-y_{h}\right) \lambda_{S \mid h}\left(1-x_{h h}^{*}\right)\right)=c .
$$

In this range of parameter values and rates of reporting suspicions, controlling disease is worthwhile if a non-reporter may become infected by the other agent, but is not worthwhile if the non-reporter faces no disease transmission risk. As a result, randomization between putting in disease control effort and shirking is the first-best response that sustains the equilibrium. Finally, if the cost of disease control is sufficiently small, non-reporters put in the disease control effort even though the risk of disease transmission is small or nil.

\footnotetext{
9 The derivation of (5) is provided in "Appendix A".
} 


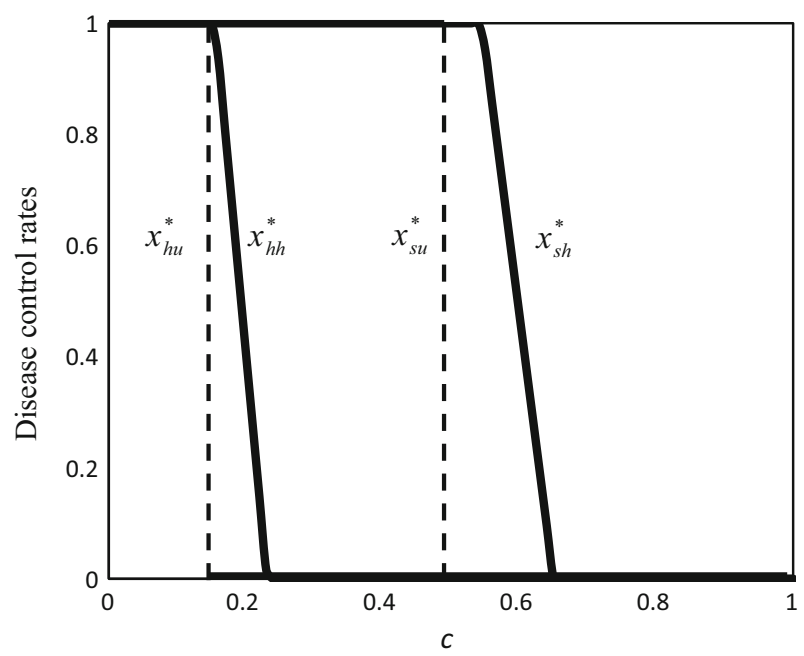

Fig. 2 Disease control rates as functions of $c$ for $y_{s}=y_{h}=0, \alpha=0.3, \beta=0.7, \delta=1$

For $y_{s}=y_{h}=0$, the equilibrium disease control efforts of non-reporters and untested reporters of suspicions as functions of the cost of disease control are depicted by solid lines (with points of discontinuity of $x_{s u}^{*}$ and $x_{h u}^{*}$ shown by dashed lines) in Fig. 2. Non-reporters tend to control the disease more often than untested reporters, but, as the reporting rates, $y_{s}$ and $y_{h}$, increase the gap between the disease control efforts of non-reporters and untested reporters shrinks. In a model with random testing at capacity, $y_{s}=y_{h}=1$, the equilibrium disease control efforts of non-reporters and untested reporters coincide,

$$
x_{z h}^{*}(1,1)=x_{z u}^{*}=\left\{\begin{array}{ll}
0, & \text { if } c>\lambda_{S \mid z} \\
1, & \text { if } c \leq \lambda_{S \mid z}
\end{array} \text { for } z \in\{s, h\},\right.
$$

because an untested agent (non-reporter or reporter) faces zero risk of disease transmission.

The equilibrium rates of disease control efforts in (5) can be rewritten in a more compact form:

$$
\begin{gathered}
x_{s h}^{*}\left(y_{h}, y_{s} ; \alpha, \beta, \delta, c\right) \\
=\min \left[\max \left[1-\frac{c-\lambda_{S \mid s}-\left(1-\lambda_{S \mid s}\right) \delta(1-g) \lambda_{S \mid h}\left(1-y_{h}\right)}{\left(1-\lambda_{S \mid s}\right) \delta g \lambda_{S \mid s}\left(1-y_{s}\right)}, 0\right], 1\right], \\
x_{h h}^{*}\left(y_{h}, y_{s} ; \alpha, \beta, \delta, c\right)=\min \left[\max \left[1-\frac{c-\lambda_{S \mid h}}{\left(1-\lambda_{S \mid h}\right) \delta(1-g) \lambda_{S \mid h}\left(1-y_{h}\right)}, 0\right], 1\right] .
\end{gathered}
$$

The following comparative statics results are all intuitive and follow directly from $\left(5^{\prime}\right)$. For given parameter values and the probabilities with which agents report suspi- 
cions, the disease control rate of a non-reporter (1) is weakly greater for a symptomatic non-reporter than asymptomatic non-reporter, $x_{s h}^{*} \geq x_{h h}^{*}$, (2) decreases with an increase in the cost of disease control, $x_{z h}^{*}(., c) \geq x_{z h}^{*}\left(., c^{\prime}\right)$ for $c<c^{\prime}$, (3) increases with an increase in disease incidence or infectiousness, $x_{z h}^{*}(., \alpha) \leq x_{z h}^{*}\left(., \alpha^{\prime}\right)$ for $\alpha<\alpha^{\prime}$ and $x_{z h}^{*}(., \delta) \leq x_{z h}^{*}\left(., \delta^{\prime}\right)$ for $\delta<\delta^{\prime},(4)$ becomes more spread out as the accuracy of signals increases, $x_{s h}^{*}(., \beta) \leq x_{s h}^{*}\left(., \beta^{\prime}\right)$ and $x_{h h}^{*}(., \beta) \geq x_{h h}^{*}\left(., \beta^{\prime}\right)$ for $\beta<\beta^{\prime}$, and (5) decreases with increases in the probabilities with which the agents demand to be tested, $x_{z h}^{*}\left(y_{s}, y_{h},.\right) \geq x_{z h}^{*}\left(y_{s}^{\prime}, y_{h}^{\prime},.\right)$ for $y_{h} \leq y_{h}^{\prime}$ and $y_{s} \leq y_{s}^{\prime}$. Property (5) can be explained as follows. When it is more likely that the agents report suspicions, an agent who does not report suspicions is less likely to become infected by another agent. This reduces the incremental payoff from exerting disease control efforts for agents who do not report suspicions. Thus, the disease control rates decrease in order to restore equilibrium. Properties (1)-(4) can be explained similarly.

\subsection{Agent who tested negative}

An agent who tested negative can only become sick at the end of the game if she is infected by the untested agent. Although a tested agent does not directly observe the other agent's symptoms or report, she can update her beliefs about the untested agent's private information, $\sigma_{H}^{*}$, based on the equilibrium reporting strategies and the fact that she was selected for testing, where $\sigma_{H}^{*}=\sigma_{S}^{*}$ since the initial disease states are drawn independently across agents. In order to calculate the probability that an untested agent infects an initially uninfected, tested agent, we need to account for the fact that the disease control strategy of the untested agent depends on whether she is symptomatic or asymptomatic, and whether she reported suspicions or not.

Using the fact that the untested agent, who observes signal $z$ and reports suspicions with probability $y_{z}$, is infectious with probability $\lambda_{S \mid z}\left[\left(1-y_{z}\right)\left(1-x_{z h}^{*}\right)+\frac{1}{2} y_{z}(1-\right.$ $x_{z u}^{*}$ )], we can calculate the conditional probability that the untested agent is infectious, $R_{t}\left(y_{s}, y_{h}\right)$, as follows:

$$
\begin{aligned}
& R_{t}\left(y_{s}, y_{h}\right) \equiv \sigma_{H}^{*} \lambda_{S \mid s} \frac{\left(1-y_{s}\right)\left(1-x_{s h}^{*}\right)+\frac{1}{2} y_{S}\left(1-x_{s u}^{*}\right)}{1-\frac{1}{2} y_{S}} \\
& \quad+\left(1-\sigma_{H}^{*}\right) \lambda_{S \mid h} \frac{\left(1-y_{h}\right)\left(1-x_{h h}^{*}\right)+\frac{1}{2} y_{h}\left(1-x_{h u}^{*}\right)}{1-\frac{1}{2} y_{h}} \\
& =\frac{g \lambda_{S \mid s}\left[\left(1-y_{s}\right)\left(1-x_{s h}^{*}\right)+\frac{1}{2} y_{s}\left(1-x_{s u}^{*}\right)\right]+(1-g) \lambda_{S \mid h}\left[\left(1-y_{h}\right)\left(1-x_{h h}^{*}\right)+\frac{1}{2} y_{h}\left(1-x_{h u}^{*}\right)\right]}{g\left(1-y_{s}\right)+(1-g)\left(1-y_{h}\right)+\frac{1}{2}\left(g y_{s}+(1-g) y_{h}\right)} \\
& \leq \frac{g\left(1-\frac{1}{2} y_{s}\right) \lambda_{S \mid s}\left(1-x_{s u}^{*}\right)+(1-g)\left(1-\frac{1}{2} y_{h}\right) \lambda_{S \mid h}\left(1-x_{h u}^{*}\right)}{1-\frac{1}{2} f\left(y_{s}, y_{h}\right)} \\
& \leq \max \left[\lambda_{S \mid s}\left(1-x_{s u}^{*}\right), \lambda_{S \mid h}\left(1-x_{h u}^{*}\right)\right]<c,
\end{aligned}
$$

where $f\left(y_{s}, y_{h}\right) \equiv g y_{s}+(1-g) y_{h}$ is the ex ante probability that an agent reports suspicions. The first inequality follows because, by (2) and (5), a non-reporter is more cautious than an untested reporter, $x_{z h}^{*} \geq x_{z u}^{*}$. The second inequality follows because 
the coefficients on $\lambda_{S \mid z}\left(1-x_{z u}^{*}\right)$ are conditional probabilities that add up to one. The third inequality follows by substituting $x_{z u}^{*}=1_{c \leq \lambda_{S \mid z}}$.

Because the probability that a tested, initially uninfected agent becomes infected in period 1 is smaller than the cost of the disease control effort, the equilibrium disease control strategy for a tested agent is given by (recall that the case with $\theta_{i, 0}=S(=1)$ was considered above in problem (1))

$$
x_{\theta}^{*}=\left\{\begin{array}{ll}
0, & \text { if } \theta=H \\
1, & \text { if } \theta=S
\end{array} .\right.
$$

The strategy in (7) is optimal for a tested agent with $\theta_{i, 0}=H(=0)$ because an untested reporter or non-reporter will control disease whenever the likelihood of her being initially infected is sufficiently great. Therefore, for a tested agent, who is uninfected in period 0 , the likelihood of becoming infected in period 1 is too small to warrant incurring the cost of disease control.

Summarizing,

Lemma 1 The equilibrium posterior beliefs $\sigma_{H}^{*}, \sigma_{S}^{*}, \sigma_{h}^{*}, \sigma_{s u}^{*}$ are given in the text, and disease control rates are given by (1), (2), (5), and (7), where $1=x_{S}^{*} \geq x_{z h}^{*} \geq x_{z u}^{*} \geq$ $x_{H}^{*}=0, z \in\{s, h\}$. The equilibrium interim (just after the test has been allocated) conditional expected losses for different types of agents are given by

$U_{z t}^{*}\left(y_{s}, y_{h}\right) \equiv \lambda_{S \mid z} c+\left(1-\lambda_{S \mid z}\right) \delta R_{t}\left(y_{s}, y_{h}\right)$,

$U_{z u}^{*} \equiv \min \left[\lambda_{S \mid z}, c\right]$,

$U_{z h}^{*}\left(y_{s}, y_{h}\right)$

$\equiv\left\{\begin{array}{ll}\min \left[\lambda_{S \mid s}+\left(1-\lambda_{S \mid s}\right) \delta\left(g\left(1-y_{s}\right) \lambda_{S \mid s}+(1-g)\left(1-y_{h}\right) \lambda_{S \mid h}\right), c\right], & \text { if } z=s \\ \min \left[\lambda_{S \mid h}+\left(1-\lambda_{S \mid h}\right) \delta(1-g)\left(1-y_{h}\right) \lambda_{S \mid h}, c\right], & \text { if } z=h\end{array}\right.$.

The payoffs (that is, the expected losses due to the cost of disease control or infection) for each type of agent with signal $z$ : tested reporter (with subscript " $z t$ "), untested reporter (with subscript " $z u$ "), and non-reporter (with subscript " $z h$ "), are obtained by substituting the equilibrium disease control strategies in (1), (2), (5), and (7) into the loss function for the respective type, and aggregating over all possible outcomes.

To understand how the expected loss of an agent with signal $z$ who is selected for testing, $U_{z t}^{*}$, is determined, consider the following. A tested agent controls the disease at cost $c$ whenever she is initially infected, which happens with probability $\lambda_{S \mid z}$. With the complimentary probability, $1-\lambda_{S \mid z}$, she is initially uninfected. Then she optimally remains exposed and becomes infected by the untested agent with probability $\delta R_{t}\left(y_{s}, y_{h}\right)$.

The calculation of the expected loss incurred by an untested agent who reported suspicions, $U_{z u}^{*}$, is particularly simple, because, having observed that she was not selected for testing, she learns that the other agent is tested with probability one. Therefore, she is only concerned with being infected in the beginning of the game and chooses whether or not to control the disease by comparing $c$ with $\lambda_{S \mid z}$.

The expected loss of an agent who did not report suspicions, $U_{z h}^{*}$, is also limited by the cost of the disease control effort. However, her expected returns to disease control 
are greater compared with the untested reporter because a non-reporter does not know whether the other agent reported suspicions (see Fig. 2 for an illustration). In the equilibrium of the post-testing disease control subgame, a symptomatic non-reporter prefers to put in the disease control effort whenever the sum of risks of being initially infected and becoming infected by a symptomatic or asymptomatic non-reporter who does not control the disease is greater than the cost of disease control:

$$
\hat{c}_{s} \equiv \lambda_{S \mid s}+\left(1-\lambda_{S \mid s}\right) \delta\left[g\left(1-y_{s}\right) \lambda_{S \mid s}+(1-g)\left(1-y_{h}\right) \lambda_{S \mid h}\right]>c .
$$

In contrast, when deciding whether or not to put in the disease control effort, in equilibrium an asymptomatic non-reporter is only concerned with disease transmission from another asymptomatic non-reporter. This asymmetry can be explained as follows. If the cost is too great for a symptomatic non-reporter to control the disease, then this is also true for an asymptomatic non-reporter. On the other hand, if the cost is small enough for a symptomatic, but not for an asymptomatic, non-reporter to prefer to control the disease, only an asymptomatic non-reporter is potentially infectious in equilibrium. As a result, the threshold cost such that disease control is optimal for an asymptomatic non-reporter is smaller than $\hat{c}_{s}$ :

$$
\hat{c}_{S}>\hat{c}_{h} \equiv \lambda_{S \mid h}+\left(1-\lambda_{S \mid h}\right) \delta(1-g)\left(1-y_{h}\right) \lambda_{S \mid h}>c .
$$

It is also worth pointing out that the post-test-assignment expected losses of a given agent are functions of the signal-contingent reporting rates adopted by the other agent, $y_{s}$ and $y_{h}$, if an agent is selected for testing, $U_{z t}^{*}\left(y_{s}, y_{h}\right)$, or did not report suspicions, $U_{z h}^{*}\left(y_{s}, y_{h}\right)$.

\section{Reporting decisions}

Substituting the period 1 expected payoffs in Lemma 1, an equilibrium pair of probabilities $\left(y_{s}^{*}, y_{h}^{*}\right)$ with which agents report suspicions solves the following system of equations

$$
\begin{aligned}
& y_{z}^{*} \in \underset{y_{z}}{\arg \min } y_{z}\left[\left(1-\frac{1}{2} f\left(y_{s}^{*}, y_{h}^{*}\right)\right) U_{z t}^{*}\left(y_{s}^{*}, y_{h}^{*}\right)+\frac{1}{2} f\left(y_{s}^{*}, y_{h}^{*}\right) U_{z u}^{*}\right] \\
& \quad+\left(1-y_{z}\right) U_{z h}^{*}\left(y_{s}^{*}, y_{h}^{*}\right)
\end{aligned}
$$

for $z \in\{s, h\}$. Because each agent takes the other agent's conditional probabilities of reporting suspicions, $y_{s}^{*}$ and $y_{h}^{*}$, as given, the optimality conditions that in equilibrium must be satisfied simultaneously are given by

$$
\left[1-\frac{1}{2} f\left(y_{s}^{*}, y_{h}^{*}\right)\right] U_{z t}^{*}\left(y_{s}^{*}, y_{h}^{*}\right)+\frac{1}{2} f\left(y_{s}^{*}, y_{h}^{*}\right) U_{z u}^{*}-U_{z h}^{*}\left(y_{s}^{*}, y_{h}^{*}\right)=(>,<) 0
$$

as $y_{z}^{*} \in(0,1)\left(y_{z}^{*}=0\right.$, or $\left.y_{z}^{*}=1\right)$ for $z \in\{s, h\}$.

The solution to system of Eq. (9) that determines the equilibrium probabilities with which the agents report suspicions, $y_{s}^{*}$ and $y_{h}^{*}$, that is, the fixed point of the map defined by (8), is completely characterized in Proposition 1. 
Proposition 1 The equilibrium probabilities with which an agent reports suspicions are given by

$$
\left(y_{s}^{*}, y_{h}^{*}\right)= \begin{cases}(1,1), & \text { if } c \leq c_{1} \\ \left(1, \hat{y}_{h}(c)\right), & \text { if } c_{1}<c<c_{2} \\ (1,0), & \text { if } c_{2} \leq c \leq c_{3} \\ \left(\hat{y}_{s}(c), 0\right), & \text { if } c>c_{3}\end{cases}
$$

where

$$
\begin{aligned}
& \hat{y}_{h}(c) \equiv \frac{\lambda_{S \mid h}(1-c)\left(1-\frac{1}{2} g\right)-\frac{1}{2} \delta\left(1-\lambda_{S \mid h}\right) \lambda_{S \mid s} g 1_{c>\lambda_{S \mid s}}}{\frac{1}{2} \lambda_{S \mid h}(1-g)\left[\delta\left(1-\lambda_{S \mid h}\right)+1-c\right]} \in(0,1) \text { for } c_{1}<c<c_{2}, \\
& \hat{y}_{S}(c) \equiv \frac{2(1-c)}{g\left[\delta\left(1-\lambda_{S \mid s}\right)+1-c\right]} \in(0,1) \text { for } c>c_{3}, \\
& c_{1}=\left\{\begin{array}{ll}
1-\left(1-\lambda_{S \mid h}\right) \delta(1-g), & \text { if } \lambda_{S \mid s} \geq 1-\left(1-\lambda_{S \mid h}\right) \delta(1-g) \\
\lambda_{S \mid s}, & \text { if } 1-\left(1-\lambda_{S \mid h}\right) \delta\left(\frac{g \lambda_{S \mid s}}{\lambda_{S \mid h}}+1-g\right) \leq \lambda_{S \mid s}<1-\left(1-\lambda_{S \mid h}\right) \delta(1-g) \\
1-\left(1-\lambda_{S \mid h}\right) \delta\left(\frac{g \lambda_{S \mid s}}{\lambda_{S \mid h}}+1-g\right), \quad \text { if } \lambda_{S \mid s}<1-\left(1-\lambda_{S \mid h}\right) \delta\left(\frac{g \lambda_{S \mid s}}{\lambda_{S \mid h}}+1-g\right)
\end{array},\right. \\
& c_{2}=\left\{\begin{array}{ll}
1-\frac{\delta g \lambda_{S \mid s}\left(1-\lambda_{S \mid h}\right)}{(2-g) \lambda_{S \mid h}}, & \text { if } 1-\frac{\delta g \lambda_{S \mid s}\left(1-\lambda_{S \mid h}\right)}{(2-g) \lambda_{S \mid h}}>\lambda_{S \mid s} \\
\lambda_{S \mid s}, & \text { if } 1-\frac{\delta g \lambda_{S \mid s}\left(1-\lambda_{S \mid h}\right)}{(2-g) \lambda_{S \mid h}} \leq \lambda_{S \mid s}
\end{array},\right. \text { and } \\
& c_{3}= 1-\frac{\delta g\left(1-\lambda_{S \mid s}\right)}{2-g} .
\end{aligned}
$$

Furthermore, $y_{s}^{*}$ and $y_{h}^{*}$ are non-increasing in $c$ and $\delta$, and are non-decreasing in $\alpha$, $y_{s}^{*}$ is non-decreasing in $\beta$.

Proof of Proposition 1 The proof is in "Appendix B".

In equilibrium, testing always occurs with positive probability, that is, an equilibrium with $\left(y_{s}^{*}, y_{h}^{*}\right)=(0,0)$ does not exist. This happens because agent $i$ takes into account that reporting suspicions, given that agent $j$ never reports suspicions, will have no effect on agent $j$ 's disease control effort (and thus will not increase agent $j$ 's infectiousness) but will allow agent $i$ to make an informed disease control decision. The inverse relationship between the disease control cost and the rates of reporting suspicions (increasing the agent/location's testing priority) can be intuitively explained as follows. As the cost of disease control increases, the option of not controlling the disease becomes more attractive. As a result, the agents more often remain exposed and are more willing to give up the testing capacity in order to minimize the chance of getting infected by the other agent.

For $c<c_{2},\left(c_{2}<c<c_{3}\right.$, or $\left.c>c_{3}\right)$, there is over-reporting (truthful reporting or under-reporting) of symptoms, respectively, in the sense that in equilibrium agents without symptoms report suspicions with positive probability (only agents with symptoms report suspicions, or agents with symptoms sometimes do not report suspicions). Furthermore, it can be verified that the threshold values of the cost of disease control that determine whether symptoms are reported too often or too rarely satisfy 


$$
\lambda_{S \mid h}<c_{1} \leq c_{2}<c_{3}<1 \text { and } \lambda_{S \mid s} \leq c_{2}
$$

The intervals of disease control costs such that in equilibrium testing is random (that is, both types of agents report suspicions with probability one) or some over-reporting occurs (that is, asymptomatic agents report suspicions with probability greater than zero), $\left(0, c_{1}\right)$ and $\left(0, c_{2}\right)$, respectively, expand as the disease incidence, $\alpha$, increases and the disease infectiousness, $\delta$, decreases. As the precision of private signals, $\beta$, increases, the interval $\left(0, c_{2}\right)$ contracts when $\beta$ is sufficiently small, and expands once $\beta$ becomes sufficiently great. The effects of $\alpha, \beta, \delta$ on the interval of disease control costs where partial over-reporting occurs, $\left(c_{1}, c_{2}\right)$, are also ambiguous. The interval of disease control costs where under-reporting occurs, $\left(c_{3}, 1\right)$ contracts as $\alpha$ and $\beta$ increase and $\delta$ decreases. The interval of disease control costs where truthful reporting occurs, $\left[c_{2}, c_{3}\right]$, contracts with an increase in $\alpha$ provided that $\beta$ is sufficiently great. However, the effects of increases in $\beta$ and $\delta$ on $\left[c_{2}, c_{3}\right]$ are ambiguous as Fig. 3 illustrates.

Figure 4 shows how the equilibrium rates of reporting suspicions by symptomatic and asymptomatic agents respond to an increase in the cost of disease control, where $y_{h}^{*}$ is depicted by a dashed line, and $y_{s}^{*}$ is a solid line. A symptomatic agent is more likely to report suspicions because she is relatively more concerned with the possibility of being initially infected than becoming infected in the future relative to an asymptomatic agent. The equilibrium rates of reporting suspicions (weakly) decrease with an increase in $c$ because, all else equal, as the cost of disease control increases the equilibrium disease control efforts (weakly) decrease which increases the probability of becoming infected by an untested agent.

For $1-\frac{\delta g \lambda_{S \mid s}\left(1-\lambda_{S \mid h}\right)}{(2-g) \lambda_{S \mid h}}>\lambda_{S \mid s}, y_{h}^{*}$ as a function of the cost of disease control exhibits a jump discontinuity at $c=\lambda_{S \mid s}$, where

$$
\lim _{c \uparrow \lambda_{S \mid s}} y_{h}^{*}>\lim _{c \downarrow \lambda_{S \mid s}} y_{h}^{*} .
$$

This happens because the equilibrium probability with which a symptomatic, untested reporter of suspicions exerts the disease control effort is discontinuous at $c=\lambda_{S \mid s}$, where

$$
\lim _{c \uparrow \lambda_{S \mid s}} x_{s u}^{*}=1>0=\lim _{c \downarrow \lambda_{S \mid s}} x_{s u}^{*} .
$$

This drop in the disease control effort by a symptomatic, untested reporter of suspicions means that an average untested agent becomes much more infectious, which makes it optimal for an asymptomatic agent to report suspicions much less frequently. On the other hand, the probability with which an agent with symptoms reports suspicions, $y_{s}^{*}$, is a continuous function of $c$. The reason is that in equilibrium with $y_{s}^{*} \in(0,1)$ there is no switch from full disease control to no effort as the disease control cost increases because a symptomatic, untested reporter of suspicions chooses $x_{s u}^{*}=0$ and an asymptomatic agent never reports suspicions for $c>c_{3}>c_{2} \geq \lambda_{S \mid s}$.

Before we consider in more detail the economic forces behind the reporting decisions, we discuss the assumption that an agent effectively observes the other agent's 


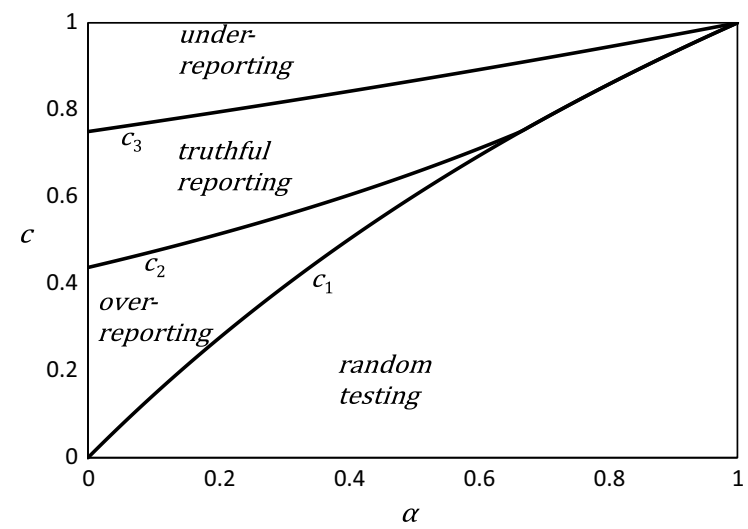

(a)

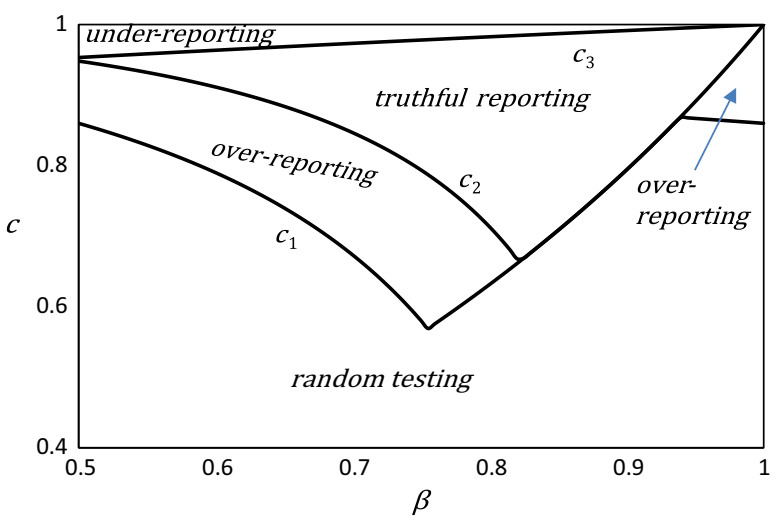

(b)

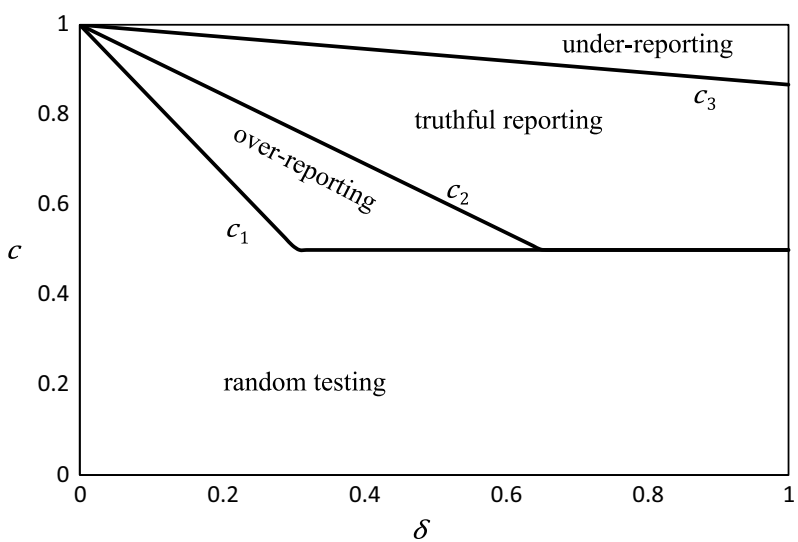

(c)

Fig. 3 Threshold values of the disease control costs as functions of $\alpha, \beta$, and $\delta$, $\mathbf{a} c_{2}$ and $c_{3}$ as functions of $\alpha$ for $\beta=0.6, \delta=1, \mathbf{b} c_{2}$ and $c_{3}$ as functions of $\beta$ for $\alpha=0.3, \delta=1, \mathbf{c} c_{2}$ and $c_{3}$ as functions of $\delta$ for $\alpha=0.3, \beta=0.7$ 


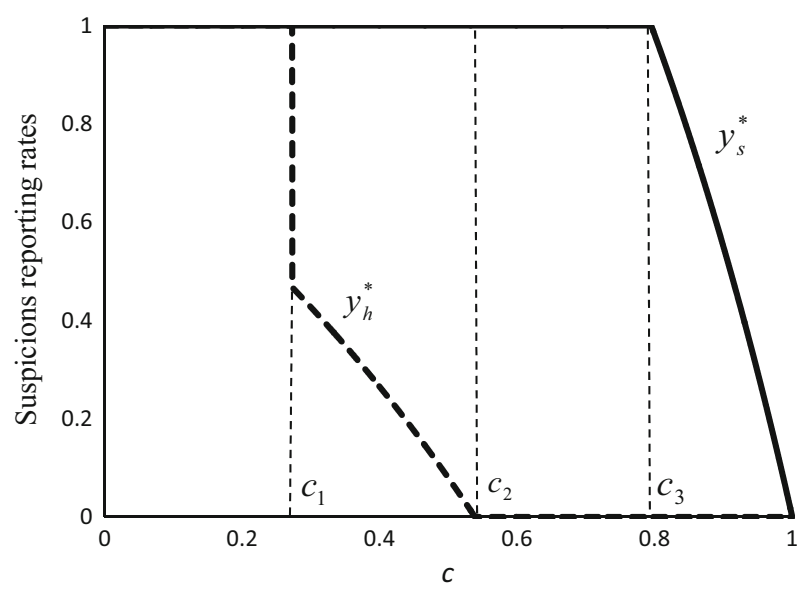

Fig. 4 Equilibrium rates of reporting suspicions as functions of the cost of disease control for $\alpha=0.2, \beta=$ $0.6, \delta=1$

report and testing status only when she is not selected for testing upon requesting it. Consider a model where the reports $r_{A}$ and $r_{B}$ (and test allocation $T$ ) are public information. If both agents report suspicions, $\left(r_{A}, r_{B}\right)=(s, s)$, the equilibrium disease control rates of the tested and untested reporter, $x_{\theta}^{* *}=x_{\theta}^{*}$ and $x_{z u}^{* *}=x_{z u}^{*}$, do not change because, as in the model with private reporting, a tested reporter controls disease only if she is initially infected and the untested reporter takes into account that the other agent is not infectious. If neither of the agents reports suspicions, $\left(r_{A}, r_{B}\right)=(h, h)$, the agents control disease more often compared with the model with private reporting and testing, $x_{z h h}^{* *} \geq x_{z h}^{*}$, where the subscript $z h h$ denotes all information available to a non-reporter, $\left(z_{i}, r_{A}, r_{B}\right)$ when reporting is public. In this case, all else equal, the probability of becoming infected by the other agent,

$$
\begin{aligned}
& \frac{g\left(1-y_{s}\right) \lambda_{S \mid s}\left(1-x_{s h h}^{* *}\right)+(1-g)\left(1-y_{h}\right) \lambda_{S \mid h}\left(1-x_{h h h}^{* *}\right)}{g\left(1-y_{s}\right)+(1-g)\left(1-y_{h}\right)} \\
& \quad>g\left(1-y_{s}\right) \lambda_{S \mid s}\left(1-x_{s h h}^{* *}\right)+(1-g)\left(1-y_{h}\right) \lambda_{S \mid h}\left(1-x_{h h h}^{* *}\right),
\end{aligned}
$$

increases compared with that in (3), and the disease control rates must rise to restore equilibrium.

If one agent reports and the other one does not report suspicions $\left(r_{A}, r_{B}\right) \in$ $\{(s, h),(h, s)\}$, the disease control strategy of the tested agent, $x_{\theta}$, does not change. As before, an agent who tested positive strictly prefers to exert effort, and an agent who tested negative strictly prefers to remain susceptible because the non-reporter controls disease whenever it is privately optimal. The probability that a reporter becomes infected by a non-reporter is again smaller than the cost of disease control:

$$
\begin{array}{r}
\delta\left[\sigma_{H} \lambda_{S \mid s}\left(1-x_{s h s}^{* *}\right)+\left(1-\sigma_{H}\right) \lambda_{S \mid h}\left(1-x_{h h s}^{* *}\right)\right] \\
\quad \leq \delta \max \left[\lambda_{S \mid s}\left(1-x_{s h s}^{* *}\right), \lambda_{S \mid h}\left(1-x_{h h s}^{* *}\right)\right] \leq c,
\end{array}
$$


for any belief of the initially uninfected reporter about the non-reporter's type, $\sigma_{H}$. However, the non-reporter controls disease less often compared with the model with private reporting since she knows that there is no risk of disease transmission from the other agent, $x_{z h s}^{* *}=x_{z u}^{*} \leq x_{z h}^{*}$. While public reporting has a positive effect on the disease control efforts in some reporting outcomes and a negative effect in other reporting outcomes, on average, the disease control efforts of untested non-reporters, and hence, the reporting rates are similar whether reporting and testing are private or public. ${ }^{10}$

\subsection{Information and externality effects of testing}

The intuition behind Proposition 1 can be explained by identifying what we characterize as information and externality effects of testing. When deciding whether or not to report suspicions and demand a test, an agent with signal $z$ weighs the value of the option to control disease as needed that is generated by knowing her initial disease state (the "information effect") against the risk of becoming infected by an untested reporter of suspicions (the "externality effect"):

$$
\begin{aligned}
& \min \left[\lambda_{S \mid z}(1-c),\left(1-\lambda_{S \mid z}\right) c\right] \\
& \text { option value generated by information about } \theta_{i, 0} \\
& =(<,>) \underset{\begin{array}{c}
\text { probability that an agent } \\
\text { is initially uninfected }
\end{array}}{\left(1-\lambda_{S \mid z}\right)} \quad \delta \frac{\frac{1}{2}\left(g y_{s}^{*} \lambda_{S \mid s} 1_{c>\lambda_{S \mid s}}+(1-g) y_{h}^{*} \lambda_{S \mid h} 1_{c>\lambda_{S \mid h}}\right)}{1-\frac{1}{2}\left(g y_{s}^{*}+(1-g) y_{h}^{*}\right)} \\
& \begin{array}{c}
\text { risk for a tested agent of becoming infected } \\
\text { by an untested reporter of suspicions }
\end{array}
\end{aligned}
$$

as $y_{z}^{*} \in(0,1)\left(y_{z}^{*}=0\right.$, or $\left.y_{z}^{*}=1\right)$ for $z \in\{s, h\}$.

The "information effect" is the option value of diagnosis that arises because an agent can avoid over-exerting or under-exerting disease control effort depending on whether the default choice is to control or not to control disease: $\min \left[\lambda_{S \mid z}, c\right]-\lambda_{S \mid z} c=$ $\min \left[\lambda_{S \mid z}(1-c),\left(1-\lambda_{S \mid z}\right) c\right]$.

Learning one's own initial disease state also means that the other agent, provided she reported suspicions, is not tested and remains infectious unless disease control is privately optimal for an untested reporter (the "externality effect"). Note that an agent's (say agent $A$ 's) decision to report suspicions has no effect on the disease control rate of agent $B$ if agent $B$ does not report suspicions because agent $B$ never finds out whether agent $A$ has been tested. As a result, when deciding whether or not to report suspicions, an agent only needs to take into account the expected decrease in the disease control rate of an untested agent who reported suspicions. The incremental probability that the other agent (agent $B$ ) is infectious due to the agent in question (agent $A$ ) getting tested is given by

\footnotetext{
10 Another complication that arises in the model with public reporting is the need to specify out-ofequilibrium beliefs in equilibrium where both types demand testing with probability one. In the model with private reporting and test allocation, there is no need to specify the out-of-equilibrium beliefs of an agent who demands a test and does not get tested in equilibrium where agents never demand testing, because a tested agent of either type is not infectious.
} 


$$
\begin{aligned}
& \underset{R_{t}\left(y_{s}^{*}, y_{h}^{*}\right)}{\begin{array}{c}
\text { conditional probability that an untested agent } \\
\text { (reporter or non-reporter) is infectious }
\end{array}}-\frac{g \lambda_{S \mid s}\left(1-y_{s}^{*}\right)\left(1-x_{s h}^{*}\right)+(1-g) \lambda_{S \mid h}\left(1-y_{h}^{*}\right)\left(1-x_{h h}^{*}\right)}{g\left(1-y_{s}^{*}\right)+(1-g)\left(1-y_{h}^{*}\right)+\frac{1}{2}\left(g y_{s}^{*}+(1-g) y_{h}^{*}\right)} \\
& \text { conditonal probability that a non-reporter is infectious } \\
& =\frac{\frac{1}{2}\left[g y_{s}^{*} \lambda_{S \mid s} 1_{c>\lambda}+(1-g) y_{h}^{*} \lambda_{S \mid h} 1_{\left.c>\lambda_{S \mid h}\right]}\right]}{1-\frac{1}{2}\left[g y_{s}^{*}+(1-g) y_{h}^{*}\right]} .
\end{aligned}
$$

If controlling disease is the default choice of an untested reporter of suspicions with signal $z, c \leq \lambda_{S \mid z}$, then the "information effect" is greater than the "externality effect" of testing. Reporting suspicions with probability one is optimal because the cost saving from avoiding the over-exertion of the disease control effort is greater than the risk of becoming infected by an untested reporter of suspicions. This happens because an untested reporter of suspicions controls disease whenever the probability of being initially infected is sufficiently great.

For $c>\lambda_{S \mid z}$, the option value generated by testing, $\lambda_{S \mid z}(1-c)$, arises because the agent avoids under-exerting disease control effort. Then in equilibrium some agents do not report suspicions because testing does not necessarily reduce exposure to the infection in period 0 by more than it increases exposure to disease transmission in period 1. The "information effect" of testing increases with the conditional probability of being initially infected, $\lambda_{S \mid z}$, and decreases with the cost of disease control, $c$. On the other hand, the "externality effect" of testing decreases with $\lambda_{S \mid z}$, (weakly) increases with $c$, and increases with $y_{s}^{*}$ and $y_{h}^{*}$. The "externality effect" depends positively on the rates of reporting suspicions because, all else equal, an untested reporter is less cautious and less likely to control the disease than is an (untested) non-reporter. As a result, in equilibrium (a) agents with symptoms report suspicions (weakly) more often than agents without symptoms, and (b) agents report suspicions (weakly) less often as the cost of disease control increases.

It is worth pointing out that, as is illustrated in Fig. 5, the option value of learning one's initial disease state is smaller (greater) for an agent with symptoms compared to that for an agent without symptoms if the cost of disease control is below (above) a certain threshold:

$$
\begin{aligned}
& \min \left[\left(1-\lambda_{S \mid s}\right) c, \lambda_{S \mid s}(1-c)\right] \leq(>) \min \left[\left(1-\lambda_{S \mid h}\right) c, \lambda_{S \mid h}(1-c)\right] \text { as } c \\
& \quad \leq(>) \lambda_{S \mid h} /\left(1-\lambda_{S \mid s}+\lambda_{S \mid h}\right) .
\end{aligned}
$$

Even though for $c \leq \lambda_{S \mid h} /\left(1-\lambda_{S \mid s}+\lambda_{S \mid h}\right)<\lambda_{S \mid s}$ symptomatic agents value learning about their initial disease state less than asymptomatic agents, in equilibrium symptomatic agents report suspicions with probability one because the "information effect" is greater than the "externality effect" for symptomatic agents.

Also, note that if the test provides an agent with imperfect information about her initial disease state, both the "information effect" and the "externality effect" become smaller. However, the analysis does not change qualitatively as it is still the case that, all else equal, (1) an agent prefers to make an informed disease control decision, and (2) a better informed agent is, on average, less infectious (that is, more likely to control disease if infected in period 0 ). 


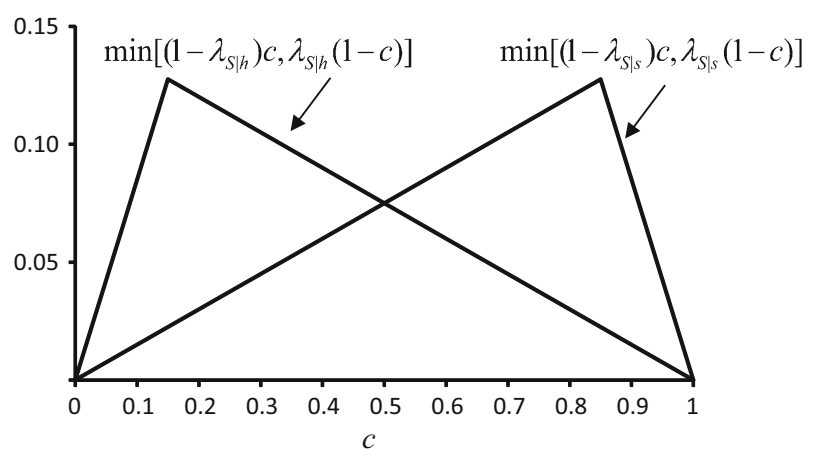

Fig. 5 Option values of diagnosis and cost of disease control for $\alpha=0.5, \beta=0.85$

\subsection{Comparative statics for $\delta, \alpha$, and $\beta$}

Consider comparative statics about the model's remaining parameters: $\delta, \alpha$, and $\beta$. As the infectiousness of the disease, $\delta$, increases, the agents report suspicions (weakly) less frequently. This happens because, all else equal, an increase in infectiousness increases the "externality effect" of undergoing testing while the "information effect" remains unchanged. As a result, as the disease infectiousness increases, the incremental payoff from testing (the difference between the left-hand side and right-hand side of (12)) decreases, and the rates at which suspicions are reported fall in order to restore equilibrium. It is worth observing that the "externality effect" of undergoing testing weakens if the allocation of the test to one agent does not imply that the other agent cannot be tested. For example, this would happen if the allocation of the testing capacity to one agent increased the probability of learning the initial disease state of that agent and decreased the probability of learning the initial disease state of the other agent. The agents would have a greater incentive to over-report symptoms if increasing one's own testing priority did not necessarily increase the infectiousness of the other agent.

The effects of the disease incidence and the precision of signals on the equilibrium rates of reporting suspicions are more complicated, because they affect both the option value of controlling disease as needed and the incremental disease transmission risk generated by testing. Consider how an increase in the disease incidence, $\alpha$, affects each term in Eq. (12). On the one hand, for $c>\lambda_{S \mid z}$, the option value generated by testing, $\lambda_{S \mid z}(1-c)$, increases with (a small increase in) $\alpha$, and the probability of remaining exposed to the disease transmission risk from the untested agents, $1-\lambda_{S \mid z}$, decreases with $\alpha$. This tends to increase the incremental payoff from getting tested. On the other hand, for fixed probabilities of reporting suspicions, $y_{s}^{*} \geq y_{h}^{*}$, the second term on the right-hand side of (12), that is, the probability of becoming infected by an untested reporter can increase or decrease with $\alpha$. Nonetheless, the disease transmission risk cannot increase too much in equilibrium because (1) untested reporters of suspicions control disease whenever $c \leq \lambda_{S \mid z}$ (which becomes more likely because $\lambda_{S \mid z}$ increases with $\alpha$ ), and (2) agents infrequently report suspicions if the probability of becoming infected by an untested reporter is sufficiently great (which reduces the effect of $\alpha$ on the incremental probability of disease transmission due to getting tested). As a result, 


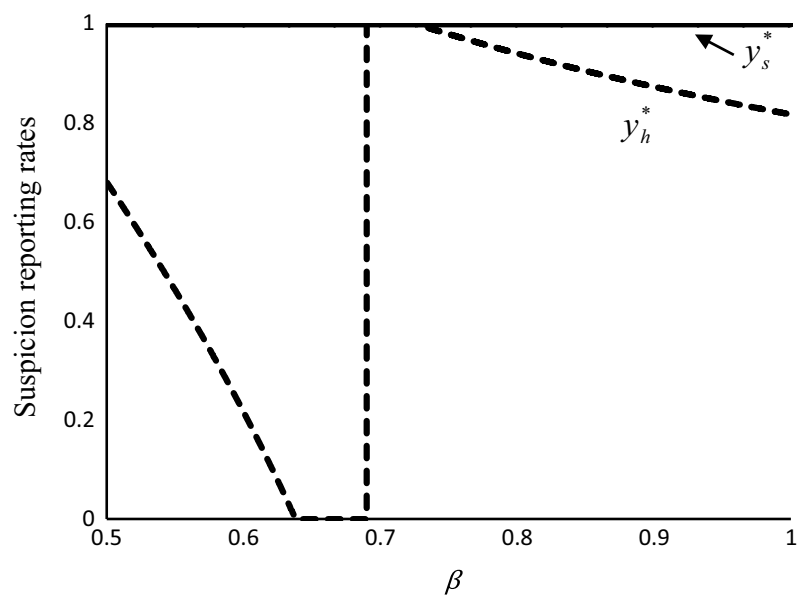

Fig. 6 Equilibrium rates of reporting suspicions and signal precision for $\alpha=0.3, c=0.5, \delta=1$

as the disease incidence, $\alpha$, increases, the incremental payoff from testing increases, and in equilibrium both types of agents report suspicions (weakly) more frequently.

The effect of the precision of signals, $\beta$, on the reporting of suspicions by agents with symptoms, $y_{s}^{*}$, is also non-negative, and can be explained similarly. ${ }^{11}$ However, the effect of $\beta$ on the probability with which agents without symptoms report suspicions, $y_{h}^{*}$, is not necessarily negative. On the one hand, for agents without symptoms the option value generated by testing, $\lambda_{S \mid h}(1-c)$, decreases and the probability of not being initially infected, $1-\lambda_{S \mid h}$, increases with $\beta$. This tends to reduce the incremental payoff from testing. On the other hand, the probability of becoming infected decreases significantly if the untested reporter of suspicions with symptoms switches from not controlling disease, $x_{s u}^{*}=1_{c \leq \lambda_{S \mid s}}=0$ to controlling disease, $x_{s u}^{*}=1_{c \leq \lambda_{S \mid s}}=1$, as private signals become more precise. An example where $y_{h}^{*}$ is a non-monotone function of $\beta$ is presented in Fig. 6, where $y_{h}^{*}$ is depicted by a dashed line, and $y_{s}^{*}=1$ is depicted by a solid line. This also explains the non-monotonic contraction and expansion of the intervals of disease control costs such that in equilibrium there is complete or partial over-reporting of symptoms in the example in Fig. 3b.

Note that the private signals may include not only observations of symptoms but also results of preliminary (perhaps self-administered) tests such as rapid diagnostic tests that are available for some infectious diseases (TDR 2008). For example, relatively simple diagnostic tools for animal infectious diseases such as Johne's disease, bovine tuberculosis and brucellosis as well as some human infectious diseases, may not yield a conclusive result without a more sophisticated analysis to correctly identify the disease (e.g., Lessells et al. 2011). In this light, a (small) improvement in a non-rationed ("off-the-shelf") testing technology can be modeled as an increase in the precision of private signals $\beta$. Our analysis suggests that better initial, rapid diagnostics may have an unintended effect of increasing the rates of reporting suspicions by individuals

\footnotetext{
11 Note that the probability of being infected in period 0 conditional on signal $s, \lambda_{S \mid s}=\alpha \beta /[\alpha \beta+(1-$ $\alpha)(1-\beta)]$, is symmetric in $\alpha$ and $\beta$.
} 
who initially tested negative even though false negatives become less likely. This may happen because those who initially tested positive will be more inclined to exercise greater care in controlling the disease, which may reduce the externality effect of one's undergoing more sophisticated (precise but rationed) diagnostic testing on overall disease control efforts.

\subsection{Truthful reporting when disease incidence is small}

How large is the set of parameters such that reporting of symptoms is truthful? As shown in Proposition 1, in equilibrium reporting is truthful if and only if $c_{2} \leq c \leq c_{3}$, which is equivalent to the following three conditions. First, a symptomatic untested reporter of suspicions prefers to remain exposed to possible infection, that is, $c>\lambda_{S \mid s}$. Second, for an asymptomatic agent the value of knowing whether or not she is initially infected is smaller than the risk of becoming infected by an untested symptomatic reporter of suspicions, $\lambda_{S \mid h}(1-c)<\left(1-\lambda_{S \mid h}\right) \frac{\frac{1}{2} \delta g \lambda_{S \mid s}}{1-\frac{1}{2} g}$. Third, for a symptomatic agent the value of knowing whether or not she is initially infected is greater than the risk of becoming infected by an untested symptomatic reporter of suspicions, $\lambda_{S \mid s}(1-c)>\left(1-\lambda_{S \mid s}\right) \frac{\frac{1}{2} \delta g \lambda_{S \mid s}}{1-\frac{1}{2} g}$.

Corollary 1 (Rare disease) Suppose that $\beta=\beta(\alpha)$ for some continuous function $\beta(\alpha) \in\left(\frac{1}{2}, 1\right)$ such that $\lim _{\alpha \rightarrow 0} \beta(\alpha)=1$ and $\lim _{\alpha \rightarrow 0} \alpha /(1-\beta(\alpha))=0 .{ }^{12}$ Then for any $c \in(0,1)$ and $\delta \in(0,1]$ there exists a threshold value $\hat{\alpha}>0$ such that $\left(y_{s}^{*}, y_{h}^{*}\right)=(1,0)$ for all $\alpha \leq \hat{\alpha}$.

Proof of Corollary 1 The proof is in "Appendix C".

Suppose that the disease is unlikely ( $\alpha$ is small) and the agents' signals are precise ( $\beta$ is large) but not too precise in the sense that even a symptomatic agent is unlikely to be infected $\left(\lambda_{S \mid s}\right.$ is small). Then for any fixed cost of disease control, $c \in(0,1)$, and degree of infectiousness, $\delta \in(0,1]$, truthful reporting tends to be optimal in equilibrium. On the one hand, an agent without symptoms tends to be relatively more concerned with the risk of disease transmission from an untested agent with symptoms (who would otherwise undergo testing) than being infected in the beginning of the game. On the other hand, an agent with symptoms tends to be relatively more concerned with avoiding the under-exertion of the disease control effort than with becoming infected by an untested agent (who is unlikely to have observed symptoms).

\subsection{Under-reporting of symptoms when only symptomatic agent can be initially infected}

It is worthwhile to consider a model where an initially healthy agent may or may not observe symptoms, $\operatorname{Pr}\left(Y_{i}=s \mid \theta_{i, 0}=H\right)=1-\operatorname{Pr}\left(Y_{i}=h \mid \theta_{i, 0}=H\right)=$ $1-\beta \in(0,1)$, but an agent who is initially infected always observes symptoms,

12 For example, function $\beta(\alpha)=1-\varepsilon \sqrt{\alpha} \in\left(\frac{1}{2}, 1\right)$ for some $\varepsilon>0$ satisfies these conditions. 
$\operatorname{Pr}\left(Y_{i}=s \mid \theta_{i, 0}=S\right)=1$. Then the conditional private beliefs about the initial disease state are given by $\lambda_{S \mid h}=0$ and $\lambda_{S \mid s}=\frac{\alpha}{\alpha+(1-\alpha)(1-\beta)}$. In this case, an asymptomatic agent knows that she is not initially infected, and does not control the disease with probability one, $x_{H}^{*}=x_{h u}^{*}=x_{h n}^{*}=0$, whether or not she is selected for testing. Hence, in any equilibrium an asymptomatic agent (at least weakly) prefers not to report suspicions in order to leave the testing capacity for the other agent. We will focus on an efficient equilibrium with $y_{h}^{*}=0$.

Consider the reporting and disease control decision of a symptomatic agent. As in the case with symmetric noise, a tested agent controls the disease in accordance with (7), and a symptomatic, untested reporter of suspicions controls the disease in accordance with (2). A symptomatic non-reporter solves

$$
x_{s h}^{*} \in \underset{x}{\arg \min }(1-x)\left(\lambda_{S \mid s}+\left(1-\lambda_{S \mid s}\right) \delta g\left(1-y_{s}\right) \lambda_{S \mid s}\left(1-x_{s h}^{*}\right)\right)+x c,
$$

where $g\left(1-y_{s}\right) \lambda_{S \mid s}\left(1-x_{s h}^{*}\right)$ is the probability that the other agent is infectious, which happens if the other agent is (1) symptomatic, and (2) does not report suspicions, and (3) does not control the disease. It can be verified that the Nash equilibrium probability of exerting disease control efforts by an asymptomatic non-reporter is given by

$$
x_{s h}^{*}\left(y_{s}, 0\right)=\left\{\begin{array}{l}
0, \quad \text { if } \lambda_{S \mid s}+\left(1-\lambda_{S \mid s}\right) \delta g\left(1-y_{s}\right) \lambda_{S \mid s}<c \\
1-\frac{c-\lambda_{S \mid s}}{\left(1-\lambda_{S \mid s}\right) \delta g\left(1-y_{s}\right) \lambda_{S \mid s}}, \quad \text { if } \lambda_{S \mid s} \leq c \leq \lambda_{S \mid s}+\left(1-\lambda_{S \mid s}\right) \delta g\left(1-y_{s}\right) \lambda_{S \mid s} \\
1, \quad \text { if } c<\lambda_{S \mid s}
\end{array} .\right.
$$

As in (9), for a given reporting strategy, $y_{s}^{*}$, a symptomatic agent decides whether to report suspicions or not based on

$$
\left(\left(1-\frac{1}{2} f\left(y_{s}^{*}, 0\right)\right) U_{s t}^{*}\left(y_{s}^{*}, 0\right)+\frac{1}{2} f\left(y_{s}^{*}, 0\right) U_{s u}^{*}-U_{s h}^{*}\left(y_{s}^{*}, 0\right)=(>,<) 0\right.
$$

as $y_{s}^{*} \in(0,1)\left(y_{s}^{*}=0\right.$, or $\left.y_{s}^{*}=1\right)$, where

$$
\begin{aligned}
U_{s t}^{*}\left(y_{s}^{*}, 0\right) & =\lambda_{S \mid s} c+\left(1-\lambda_{S \mid s}\right) \delta \frac{g\left(1-\frac{1}{2} y_{s}^{*}\right) \lambda_{S \mid s} 1_{c>\lambda_{S \mid s}}}{1-\frac{1}{2} g y_{s}^{*}}, \\
U_{s u}^{*} & =\min \left[\lambda_{S \mid s}, c\right], \\
U_{s h}^{*}\left(y_{s}^{*}, 0\right) & =\min \left[\lambda_{S \mid s}+\left(1-\lambda_{S \mid s}\right) \delta g\left(1-y_{s}^{*}\right) \lambda_{S \mid s}, c\right] .
\end{aligned}
$$

Substituting the expected losses for a tested and untested symptomatic reporter of suspicions, $U_{s t}^{*}\left(y_{s}^{*}, 0\right)$ and $U_{s u}^{*}$, and a symptomatic non-reporter, $U_{s h}^{*}\left(y_{s}^{*}, 0\right)$, Eq. (15) becomes

$$
\begin{aligned}
& \left(1-\frac{1}{2} g y_{s}^{*}\right) \lambda_{S \mid s} c+\left(1-\lambda_{S \mid s}\right) \delta g\left(1-\frac{1}{2} y_{s}^{*}\right) \lambda_{S \mid s} 1_{c>\lambda_{S \mid s}}+\frac{1}{2} g y_{s}^{*} \min \left[\lambda_{S \mid s}, c\right] \\
& -\min \left[\lambda_{S \mid s}+\left(1-\lambda_{S \mid s}\right) \delta g\left(1-y_{s}^{*}\right) \lambda_{S \mid s}, c\right]=(>,<) 0 .
\end{aligned}
$$


It can be verified that a unique solution is $y_{s}^{*}=\min \left[\hat{y}_{s}(c), 1\right]$. The equilibrium rate of reporting suspicions for a symptomatic agent as a function of the variables $c, \lambda_{S \mid s}, g, \delta$ is the same in the case with symmetric noise in private signals even though only symptomatic agents impose disease transmission risk in the model with asymmetric noise. The reason is that in both models in equilibrium a symptomatic agent who is indifferent between reporting suspicions or not, and decides to forego testing, will increase the probability that the other agent gets tested only if the other agent is also symptomatic. In the model where signal $Z_{i}=h$ is perfectly informative, in the efficient equilibrium there is no over-reporting of symptoms because asymptomatic agents cannot achieve a smaller loss by undergoing testing. As a result, reporting is truthful as long as the cost of disease control is sufficiently small. However, as in the model with symmetric noise, under-reporting of symptoms occurs if the cost of disease control is sufficiently great.

\section{Test subsidy/tax}

Here we study the welfare economics of limited diagnostic testing. Consider a subsidy/tax scheme under which a tested agent gets a transfer (positive or negative) $\tau$ and an untested agent gets no transfers. The optimality conditions that determine the equilibrium disease control efforts do not change, but the optimality conditions (12) that determine the equilibrium rates of reporting suspicions become

$$
\begin{aligned}
& \min \left[\left(1-\lambda_{S \mid z}\right) c, \lambda_{S \mid z}(1-c)\right]+\tau \\
& =(<,>)\left(1-\lambda_{S \mid z}\right) \frac{\frac{1}{2} \delta\left(g y_{s}^{*} \lambda_{S \mid s} 1_{c>\lambda_{S \mid s}}+(1-g) y_{h}^{*} \lambda_{S \mid h} 1_{c>\lambda_{S \mid h}}\right)}{1-\frac{1}{2}\left(g y_{s}^{*}+(1-g) y_{h}^{*}\right)}
\end{aligned}
$$

as $y_{z}^{*} \in(0,1)\left(y_{z}^{*}=0\right.$, or $\left.y_{z}^{*}=1\right)$ for $z \in\{s, h\}$. Let $y_{s}^{*}(\tau)$ and $y_{h}^{*}(\tau)$ denote the equilibrium rates of reporting suspicions, where the equilibrium without transfers is obtained by setting $\tau=0$. Because the "externality effect" of testing on the right-hand side of (17) is increasing in $y_{h}^{*}$ and $y_{s}^{*}$, the equilibrium test-demand rates $y_{s}^{*}(\tau)$ and $y_{h}^{*}(\tau)$ are also increasing in $\tau$.

First, we consider the case where truthful reporting of suspicions maximizes the expected welfare.

Lemma 2 Suppose that $c \geq \lambda_{S \mid s}+\delta\left(1-\lambda_{S \mid s}\right) \lambda_{S \mid s}$ and $g \lambda_{S \mid s}>(2-g) \lambda_{S \mid h}$. Then truthful reporting is socially optimal.

\section{Proof of Lemma 2 The proof is in "Appendix C".}

The first condition ensures that an untested agent of any type does not control the disease. As a result, the option value of information generated by testing is greater for a symptomatic agent, and an untested symptomatic agent poses a greater risk of disease transmission compared with an asymptomatic agent. The second condition, which can be equivalently rewritten as $\alpha<\left(2 \beta^{2}-1\right) /(2 \beta-1)^{2}$, is satisfied whenever the initial prevalence is not too great and private signals are sufficiently precise. Then 
completely excluding asymptomatic agents from testing in order to ensure that a symptomatic agent gets tested maximizes expected welfare.

If the first condition in Lemma 2 is satisfied then condition (17) becomes

$$
\lambda_{S \mid z}(1-c)+\tau=(<,>) \delta\left(1-\lambda_{S \mid z}\right) \frac{g y_{s}^{*} \lambda_{S \mid s}+(1-g) y_{h}^{*} \lambda_{S \mid h}}{2-g y_{s}^{*}-(1-g) y_{h}^{*}} .
$$

For $c>c_{3}$ symptoms are under-reported, $y_{h}^{*}(0)=0$ and $y_{s}^{*}(0) \in(0,1)$, in equilibrium without transfers, but truthful reporting, $\left(y_{s}^{*}(\tau), y_{h}^{*}(\tau)\right)=(1,0)$, can be achieved under a test subsidy

$$
\tau^{*}=\left(1-\lambda_{S \mid s}\right) \frac{\delta g \lambda_{S \mid s}}{2-g}-\lambda_{S \mid s}(1-c)>0 .
$$

If testing is subsidized, a symptomatic agent is indifferent between reporting suspicions or not while an asymptomatic agent strictly prefers not to report suspicions. The test subsidy just offsets the greater disease transmission risk net of the benefit of information about the initial disease incidence for a tested symptomatic agent. For $c_{2} \leq c \leq c_{3}$, transfers are not necessary to achieve truthful reporting, $\tau^{*}=0$. For $c<c_{2}$ symptoms are over-reported, $y_{h}^{*}(0) \in(0,1]$ and $y_{s}^{*}(0)=1$, in equilibrium without transfers, but truthful reporting can be achieved under a test tax

$$
\tau^{*}=\left(1-\lambda_{S \mid h}\right) \frac{\delta g \lambda_{S \mid s}}{2-g}-\lambda_{S \mid h}(1-c)<0 .
$$

If testing is taxed, an asymptomatic agent is indifferent between reporting suspicions or not while a symptomatic agent strictly prefers to report suspicions. The test tax just offsets the benefit of information about the initial disease incidence net of the greater disease transmission risk for a tested asymptomatic agent. Summarizing,

Proposition 2 Suppose that $c \geq \lambda_{S \mid s}+\delta\left(1-\lambda_{S \mid s}\right) \lambda_{S \mid s}$ and $g \lambda_{S \mid s}>(2-g) \lambda_{S \mid h}$. Then welfare is maximized under a test subsidy (no transfers, or test tax) for $c>c_{3}\left(c_{2} \leq\right.$ $c \leq c_{3}$, or $c<c_{2}$ ).

However, truthful reporting does not maximize welfare if the cost of disease control is small or symptoms are rarely observed. When only untested asymptomatic agents do not control the disease then welfare may increase if the test is allocated to an asymptomatic agent. If the symptoms are not observed often, then testing of asymptomatic agents may also increase welfare since otherwise the testing capacity will tend to be underutilized.

So far we have assumed that only agents who report suspicions can be selected for testing. This is a plausible assumption if testing is voluntary and individuals who do not report suspicions cannot be enrolled into a testing program as in the case where diagnostic testing requires visiting a medical facility or submitting a blood sample for laboratory analysis. An alternative scenario is that any agent can be selected for testing but the testing priorities are assigned based on the reports of symptoms submitted by agents. This is a plausible characterization when a veterinary official inspects 
farm animals in the course of a vaccination program or routine visits. In this case, underutilization of the testing capacity can be avoided and a schedule of transfers that are contingent on the agent's report and the outcome of the test can always implement the efficient allocation of the test. The problem of test allocation is then similar to the problem of second-degree price discriminating monopoly that offers a menu of prices and qualities (testing priorities in our case) to consumers with private valuations for the good (signals of the initial disease incidence in our case).

\section{Many agents}

Here we discuss what happens if the number of agents is greater than two. Suppose there are $N / 2 \geq 2$ identical pairs of agents $\{i, i+1\}$ with one diagnostic test per pair. The final probability that agent is infected is now given by

$$
\begin{aligned}
& \operatorname{Pr}\left(\theta_{i, 1}=S \mid \theta_{1,0}, \ldots, \theta_{N, 0}, e_{1}, \ldots, e_{N}\right) \\
& \quad=\left[\theta_{i, 0}+\delta\left(\gamma\left(1-e_{i+1}\right) \theta_{i+1,0}+\frac{1-\gamma}{N-2} \sum_{j=1, j \neq i, i+1}^{N}\left(1-e_{j}\right) \theta_{j, 0}\right)\right]\left(1-e_{i}\right),
\end{aligned}
$$

if $i$ is odd, and

$$
\begin{aligned}
& \operatorname{Pr}\left(\theta_{i, 1}=S \mid \theta_{1,0}, \ldots, \theta_{N, 0}, e_{1}, \ldots, e_{N}\right) \\
& \quad=\left[\theta_{i, 0}+\delta\left(\gamma\left(1-e_{i-1}\right) \theta_{i-1,0}+\frac{1-\gamma}{N-2} \sum_{j=1, j \neq i, i-1}^{N}\left(1-e_{j}\right) \theta_{j, 0}\right)\right]\left(1-e_{i}\right),
\end{aligned}
$$

if $i$ is even, where $\gamma \in[0,1]$ is the measure of internal (local) infectiousness and $1-\gamma$ is the measure of external (global) infectiousness. Each pair of agents $\{i, i+1\}$ for $i=1,3,5, \ldots$ is equally "distanced" from all other pairs. All other features of the model, including the procedure for allocating the test between the members of each pair $\{i, i+1\}$, remain unchanged. The previous analysis considered the case with the pure internal disease transmission, $\gamma=1$. So we now assume that $\gamma<1$.

This specification of the disease transmission risk is plausible if the constraints on diagnostic tests are more local than infectiousness in the sense that individuals who do not compete for the same diagnostic capacity can still infect each other. For example, there is little competition for testing between individuals who live near different, spatially separated diagnostic facilities if patients and medical personnel have to be physically present for a test or sample collection, and travelling to a more distant diagnostic facility is costly or access is restricted to certain groups of individuals. Then stochastic spread of an infectious disease in an area that includes several "local" diagnostic centers can be represented using Eqs. (21) and (22) above. 
The external risk (average probability) of disease transmission (from all pairs excluding the pair in question) is given by

$$
\begin{aligned}
R_{0}\left(y_{s}, y_{h}, x_{s u}, x_{s h}, x_{h u}, x_{h h}\right) \equiv & \sum_{z \in\{s, h\}} \lambda_{z} g_{z}\left[\frac{1}{2} y_{z} f\left(y_{s}, y_{h}\right)\left(1-x_{z u}\right)\right. \\
& \left.+\left(1-y_{z}\right)\left(1-x_{z h}\right)\right]
\end{aligned}
$$

where $g_{s}=g$ and $g_{h}=1-g$. As before, an agent with signal $z$ is infectious if she was infected in period 0 , and (1) she reported suspicions, was not selected for testing, and does not control the disease, which happens with probability $\frac{1}{2} y_{z} f\left(y_{s}, y_{h}\right)\left(1-x_{z u}\right) \lambda_{z}$, or (2) she did not report suspicions and does not control the disease, which happens with probability $\left(1-y_{z}\right)\left(1-x_{z h}\right) \lambda_{z}$.

In equilibrium an agent who reported suspicions but was not selected for testing now chooses the level of disease control $x_{z u}$ to minimize

$$
x_{z u}^{*} \in \underset{x}{\arg \min } x \lambda_{z}+(1-x) \delta(1-\gamma) R_{0}\left(y_{s}, y_{h}, x_{s u}^{*}, x_{s h}^{*}, x_{h u}^{*}, x_{h h}^{*}\right)
$$

The aggregate disease transmission risk faced by an agent who did not report suspicions is given by

$$
\begin{aligned}
& \gamma \sum_{z \in\{s, h\}} \lambda_{z} g_{z}\left(1-y_{z}\right)\left(1-x_{z h}\right)+(1-\gamma) R_{0} \\
& \quad=\sum_{z \in\{s, h\}} \lambda_{z} g_{z}\left[\left(1-y_{z}\right)\left(1-x_{z h}\right)+\frac{1-\gamma}{2} y_{z} f\left(y_{s}, y_{h}\right)\left(1-x_{z u}\right)\right],
\end{aligned}
$$

so that the equilibrium level of disease control chosen by a non-reporter solves

$$
\begin{aligned}
& x_{z h}^{*} \in \underset{x}{\arg \min } x \lambda_{z}+(1-x) \delta \sum_{z \in\{s, h\}} \lambda_{z} g_{z}\left[\left(1-y_{z}\right)\left(1-x_{z h}^{*}\right)\right. \\
& \left.+\frac{1-\gamma}{2} y_{z} f\left(y_{s}, y_{h}\right)\left(1-x_{z u}^{*}\right)\right] .
\end{aligned}
$$

An untested member of the pair (reporter and non-reporter) chooses a greater level of disease control when, due to limited testing, there is some residual risk of disease transmission from the other pairs,

$$
x_{z u}^{*}(\gamma) \geq x_{z u}^{*}(1)=1_{c \leq \lambda S \mid z} \text { and } x_{z h}^{*}(\gamma) \geq x_{z h}^{*}(1) .
$$

Finally, as in the model with pure internal disease transmission risk, a tested agent controls the disease if and only if she is initially infected because the abatement efforts taken by the potential transmitters reduce the risk for an initially uninfected agent below the threshold at which disease control is efficient.

As in the case with pure internal transmission risk, it can be verified that the optimality conditions that determine the equilibrium rates of reporting suspicions are given 
by $^{13}$

$$
\begin{aligned}
& \min \left[\left(1-\lambda_{S \mid z}\right) c, \lambda_{S \mid z}(1-c)\right] \\
& =(<,>)\left(1-\lambda_{S \mid z}\right) \frac{\frac{1}{2} \delta \gamma \sum_{b \in\{s, h\}} g_{b} y_{b}^{*} \lambda_{S \mid b}\left(1-x_{b u}^{*}(\gamma)\right)}{1-\frac{1}{2}\left(g_{s} y_{s}^{*}+g_{h} y_{h}^{*}\right)} .
\end{aligned}
$$

A comparison of (12) and (27) reveals that the equilibrium rates of reporting suspicions decrease with $\gamma$. In the presence of the external disease transmission risk (from the other agents with separate testing capacity) the test utilization rates are higher because each agent is less concerned with crowding out the local testing capacity. Note that there are two channels through which external disease transmission affects the rates of reporting suspicions. First, the total disease transmission risk faced by the agent in question depends less on whether that agent or the other member of the pair is selected for testing. Second, the disease control efforts of the agent who reported suspicions but was not selected for testing is greater, which reduces the risk of local (internal) disease transmission. However, as long as the degree of local (relative to global) infectiousness (risk of internal disease transmission), $\gamma$, is not too small, Propositions 1 and 2 continue to hold qualitatively in the sense that in equilibrium symptoms can be over-reported or under-reported depending on the parameter values of the model.

\section{Conclusions}

In this paper, we studied a model of detection and control of an infectious disease in the presence of limited testing and private information about symptoms. We showed that symptoms can be under-, over- or truthfully reported depending on the cost of disease control, the incidence of disease, the precision of private information, and the infectiousness of the disease. We also demonstrated that it is socially optimal to subsidize, to neither subsidize nor tax, or to tax limited diagnostic testing whenever the cost of disease control is, respectively, sufficiently great, intermediate, or sufficiently small and certain additional parametric restrictions are satisfied. Furthermore, these results hold in a setting with multiple potentially infected agents and localized testing capacities if the risk of transmission from outside is not too great.

In our analysis, we focused on the case where the effectiveness of the disease control measures is independent of the outcome of the diagnostic test as in the case of culling potentially infected animals to avoid further contamination and stop the spread of disease. However, our results regarding the equilibrium rates of reporting suspicions will continue to hold in a setting where disease control is more effective if the disease is formally identified and a more selective treatment is possible. Our model can also be extended in several additional ways. In our setting, the negative externalities arise solely due to uncertainty about initial disease incidence among the untested agents/localities. By allowing for a convex cost of disease control, we could

13 The conditions (12) and (27) are similar because the common risk of external transmission $R_{0}$ (which is independent of the test allocation within a pair of agents) in the expected payoffs from reporting suspicions and not reporting suspicions cancels out. 
study the design of a disease surveillance program when not only untested localities but also localities that test positive for infection impose negative externalities on the other localities (Olmstead and Rhode 2004). Also, by allowing localities to control their initial disease status, we could study the effects of early detection on the preventive actions. Another interesting extension would be to consider a more general private information structure and reports that are partially informative. Also left for future research is the design of a dynamic surveillance program that incentivizes reporting of clinically suspect cases and the allocation of diagnostic resources in a continuous time susceptible-infected-recovered model of an infectious disease (Anderson and May 1979).

\section{Appendix A}

\section{Derivation of the equilibrium disease control rates for agents who do not report suspicions}

Because in a Nash equilibrium of the post-testing disease control game an agent takes the disease control strategy of the other agent as given, the optimality conditions for problem (4) are given by

$$
\begin{aligned}
& \lambda_{S \mid z}+\left(1-\lambda_{S \mid z}\right) R_{h}\left(x_{s h}^{*}, x_{h h}^{*}\right) \\
& \quad=(<,>) c \text { as } x_{z h}^{*} \in(0,1)\left(x_{z h}^{*}=0, \text { or } x_{z h}^{*}=1\right) \text { for } z \in\{s, h\},
\end{aligned}
$$

where $R_{h}\left(x_{s h}^{*}, x_{h h}^{*}\right)=\delta\left[g\left(1-y_{s}\right) \lambda_{S \mid s}\left(1-x_{s h}^{*}\right)+(1-g)\left(1-y_{h}\right) \lambda_{S \mid h}\left(1-x_{h h}^{*}\right)\right]$ is the equilibrium disease transmission risk. Because

$$
\lambda_{S \mid s}+\left(1-\lambda_{S \mid s}\right) R_{h}\left(x_{s h}^{*}, x_{h h}^{*}\right)>\lambda_{S \mid h}+\left(1-\lambda_{S \mid h}\right) R_{h}\left(x_{s h}^{*}, x_{h h}^{*}\right)
$$

it follows that in equilibrium a symptomatic non-reporter of suspicions puts in a weakly greater effort than an asymptomatic non-reporter, $x_{s h}^{*} \geq x_{h h}^{*}$. Therefore, there are at most five types of possible equilibrium outcomes: (1) $\left(x_{s h}^{*}, x_{h h}^{*}\right)=(0,0),(2) x_{h h}^{*}=0$ and $0<x_{s h}^{*}<1$, (3) $\left(x_{s h}^{*}, x_{h h}^{*}\right)=(1,0)$, (4) $x_{s h}^{*}=1$ and $0<x_{h h}^{*}<1$, and (5) $\left(x_{s h}^{*}, x_{h h}^{*}\right)=(1,1)$.

Case 1 Suppose that $\left(x_{s h}^{*}, x_{h h}^{*}\right)=(0,0)$. Substituting $\left(x_{s h}^{*}, x_{h h}^{*}\right)=(0,0)$ into (A1), we find that each agent prefers not to control the disease if

$$
\lambda_{S \mid s}+\left(1-\lambda_{S \mid s}\right) \delta\left[g\left(1-y_{s}\right) \lambda_{S \mid s}+(1-g)\left(1-y_{h}\right) \lambda_{S \mid h}\right]<c .
$$

Thus, $\left(x_{s h}^{*}, x_{h h}^{*}\right)=(0,0)$ is the unique solution if and only if (A3) holds.

Case 2 Suppose that $x_{h h}^{*}=0$ and $x_{s h}^{*} \in(0,1)$. From (A1) and (A2), it follows that an asymptomatic non-reporter strictly prefers not to control the disease, while a symptomatic non-reporter is indifferent if and only if

$$
\begin{aligned}
\lambda_{S \mid s}+\left(1-\lambda_{S \mid s}\right) \delta(1-g)\left(1-y_{h}\right) \lambda_{S \mid h} \leq & c \leq \lambda_{S \mid s}+\left(1-\lambda_{S \mid s}\right) \delta\left[g\left(1-y_{s}\right) \lambda_{S \mid s}\right. \\
& \left.+(1-g)\left(1-y_{h}\right) \lambda_{S \mid h}\right] .
\end{aligned}
$$


Substituting $x_{h h}^{*}=0$ and $x_{s h}^{*} \in(0,1)$ into (A1), the optimality condition for a symptomatic non-reporter becomes

$$
\lambda_{S \mid z}+\left(1-\lambda_{S \mid z}\right) \delta\left[g\left(1-y_{s}\right) \lambda_{S \mid s}\left(1-x_{s h}^{*}\right)+(1-g)\left(1-y_{h}\right) \lambda_{S \mid h}\right]=c .
$$

The unique solution is given by

$$
x_{s h}^{*}=1-\frac{c-\lambda_{S \mid s}-\left(1-\lambda_{S \mid s}\right) \delta(1-g)\left(1-y_{h}\right) \lambda_{S \mid h}}{\left(1-\lambda_{S \mid s}\right) \delta g\left(1-y_{s}\right) \lambda_{S \mid s}} .
$$

Case 3 Suppose that $\left(x_{s h}^{*}, x_{h h}^{*}\right)=(1,0)$. Substituting $\left(x_{s h}^{*}, x_{h h}^{*}\right)=(1,0)$, it follows from (A1) that an asymptomatic non-reporter of suspicions strictly prefers not to control the disease, and a symptomatic non-reporter strictly prefers to control the disease if and only if

$$
\lambda_{S \mid h}+\left(1-\lambda_{S \mid h}\right) \delta(1-g)\left(1-y_{h}\right) \lambda_{S \mid h}<c<\lambda_{S \mid S}+\left(1-\lambda_{S \mid S}\right) \delta(1-g)\left(1-y_{h}\right) \lambda_{S \mid h} .
$$

Case 4 Suppose that $x_{s h}^{*}=1$ and $x_{h h}^{*} \in(0,1)$. From (A1), it follows that an asymptomatic non-reporter is indifferent between controlling and not controlling the disease, and a symptomatic non-reporter strictly prefers to control the disease if and only if

$$
\lambda_{S \mid h} \leq c \leq \lambda_{S \mid h}+\left(1-\lambda_{S \mid h}\right) \delta(1-g)\left(1-y_{h}\right) \lambda_{S \mid h} .
$$

Substituting $x_{s h}^{*}=1$ and $x_{h h}^{*} \in(0,1)$ into (A1), the optimality condition for an asymptomatic non-reporter becomes

$$
\lambda_{S \mid h}+\left(1-\lambda_{S \mid h}\right) \delta(1-g)\left(1-y_{h}\right) \lambda_{S \mid h}\left(1-x_{h h}^{*}\right)=c .
$$

The unique solution is given by

$$
x_{h h}^{*}=1-\frac{c-\lambda_{S \mid h}}{\left(1-\lambda_{S \mid h}\right) \delta(1-g)\left(1-y_{h}\right) \lambda_{S \mid h}} .
$$

Case 5 Suppose that $\left(x_{s h}^{*}, x_{h h}^{*}\right)=(1,1)$. Upon substituting $\left(x_{s h}^{*}, x_{h h}^{*}\right)=(1,1)$ into (A1), it follows that both types of non-reporters strictly prefer to control the disease if and only if $c<\lambda_{S \mid h}$.

\section{Appendix B}

Proof of Proposition 1 From inequality (6) in the text, it follows that

$$
U_{z t}^{*}\left(y_{s}, y_{h}\right)=\lambda_{S \mid z} c+\left(1-\lambda_{S \mid z}\right) \delta R_{t}\left(y_{s}, y_{h}\right)<\lambda_{S \mid z} c+\left(1-\lambda_{S \mid z}\right) c=c .
$$

Therefore, if $U_{z h}^{*}\left(y_{s}^{*}, y_{h}^{*}\right)=c$ then in equilibrium $y_{z}^{*}=1$ because the incremental payoff from reporting suspicions is necessarily negative (that is, an agent who reports suspicions achieves a smaller expected loss than an agent who does not report suspicions): 


$$
\begin{gathered}
P_{z}\left(y_{s}^{*}, y_{h}^{*}\right) \equiv\left[1-\frac{1}{2} f\left(y_{s}^{*}, y_{h}^{*}\right)\right] U_{z t}^{*}\left(y_{s}^{*}, y_{h}^{*}\right)+\frac{1}{2} f\left(y_{s}^{*}, y_{h}^{*}\right) U_{z u}^{*}-U_{z h}^{*}\left(y_{s}^{*}, y_{h}^{*}\right) \\
\quad<\left[1-\frac{1}{2} f\left(y_{s}^{*}, y_{h}^{*}\right)\right] c+\frac{1}{2} f\left(y_{s}^{*}, y_{h}^{*}\right) U_{z u}^{*}-c=\frac{1}{2} f\left(y_{s}^{*}, y_{h}^{*}\right)\left(U_{z u}^{*}-c\right) \leq 0
\end{gathered}
$$

where $P_{z}\left(y_{s}^{*}, y_{h}^{*}\right)$ is the incremental payoff (expected loss) from reporting suspicions.

So suppose that in equilibrium $U_{s h}^{*}\left(y_{s}^{*}, y_{h}^{*}\right)<c$ for $z \in\{s, h\}$. By Lemma 1 , it must be that $\lambda_{S \mid s} \leq U_{s h}^{*}\left(y_{s}^{*}, y_{h}^{*}\right)<c$ and $\lambda_{S \mid h} \leq U_{h h}^{*}\left(y_{s}^{*}, y_{h}^{*}\right)<c$, and the incremental payoffs from reporting suspicions become

$$
\begin{aligned}
& P_{S}\left(y_{s}^{*}, y_{h}^{*}\right)=\left(1-\frac{1}{2} f\left(y_{s}^{*}, y_{h}^{*}\right)\right)\left[\lambda_{S \mid s} c+\left(1-\lambda_{S \mid s}\right) \delta R_{t}\left(y_{s}^{*}, y_{h}^{*}\right)\right] \\
& +\frac{1}{2} f\left(y_{s}^{*}, y_{h}^{*}\right) \lambda_{S \mid s}-\lambda_{S \mid s}-\left(1-\lambda_{S \mid s}\right) \delta\left[g\left(1-y_{s}^{*}\right) \lambda_{S \mid s}+(1-g)\left(1-y_{h}^{*}\right) \lambda_{S \mid h}\right] \\
& =-\left(1-\frac{1}{2} f\left(y_{s}^{*}, y_{h}^{*}\right)\right) \lambda_{S \mid s}(1-c) \\
& +\left(1-\lambda_{S \mid s}\right) \delta\left(g \lambda_{S \mid s}\left[\left(1-y_{s}^{*}\right)\left(1-x_{s h}^{*}\right)+\frac{1}{2} y_{s}^{*}\left(1-x_{s u}^{*}\right)\right]\right. \\
& +(1-g) \lambda_{S \mid h}\left[\left(1-y_{h}^{*}\right)\left(1-x_{h h}^{*}\right)+\frac{1}{2} y_{h}^{*}\left(1-x_{h u}^{*}\right)\right] \\
& \left.-g\left(1-y_{s}^{*}\right) \lambda_{S \mid s}-(1-g)\left(1-y_{h}^{*}\right) \lambda_{S \mid h}\right) \\
& =-\left(1-\lambda_{S \mid s}\right)\left(1-\frac{1}{2} f\left(y_{s}^{*}, y_{h}^{*}\right)\right)\left(\frac{\lambda_{S \mid s}}{1-\lambda_{S \mid s}}(1-c)\right. \\
& \left.-\delta \frac{g \lambda_{S \mid s}\left(\frac{1}{2} y_{s}^{*}\left(1-x_{s u}^{*}\right)-\left(1-y_{s}^{*}\right) x_{s h}^{*}\right)+(1-g) \lambda_{S \mid h}\left(\frac{1}{2} y_{h}^{*}\left(1-x_{h u}^{*}\right)-\left(1-y_{h}^{*}\right) x_{h h}^{*}\right)}{1-\frac{1}{2} f\left(y_{s}^{*}, y_{h}^{*}\right)}\right)
\end{aligned}
$$

and

$$
\begin{aligned}
P_{h}\left(y_{s}^{*}, y_{h}^{*}\right)= & \left(1-\frac{1}{2} f\left(y_{s}^{*}, y_{h}^{*}\right)\right)\left[\lambda_{S \mid h} c+\left(1-\lambda_{S \mid h}\right) \delta R_{t}\left(y_{s}^{*}, y_{h}^{*}\right)\right] \\
& +\frac{1}{2} f\left(y_{s}^{*}, y_{h}^{*}\right) \lambda_{S \mid s}-\lambda_{S \mid h}-\left(1-\lambda_{S \mid h}\right) \delta(1-g)\left(1-y_{h}^{*}\right) \lambda_{S \mid h} \\
& =-\left(1-\frac{1}{2} f\left(y_{s}^{*}, y_{h}^{*}\right)\right) \lambda_{S \mid h}(1-c) \\
& +\left(1-\lambda_{S \mid h}\right) \delta\left(g \lambda_{S \mid s}\left[\left(1-y_{s}^{*}\right)\left(1-x_{s h}^{*}\right)+\frac{1}{2} y_{s}^{*}\left(1-x_{s u}^{*}\right)\right]\right. \\
& +(1-g) \lambda_{S \mid h}\left[\left(1-y_{h}^{*}\right)\left(1-x_{h h}^{*}\right)+\frac{1}{2} y_{h}^{*}\left(1-x_{h u}^{*}\right)\right] \\
& \left.\left.-(1-g)\left(1-y_{h}^{*}\right) \lambda_{S \mid h}\right)\right) \\
& =-\left(1-\lambda_{S \mid h}\right)\left(1-\frac{1}{2} f\left(y_{s}^{*}, y_{h}^{*}\right)\right)\left(\frac{\lambda_{S \mid h}}{1-\lambda_{S \mid h}}(1-c)\right. \\
& \left.-\delta \frac{g \lambda_{S \mid s}\left[\frac{1}{2} y_{s}^{*}\left(1-x_{s u}^{*}\right)+\left(1-y_{s}^{*}\right)\left(1-x_{s h}^{*}\right)\right]+(1-g) \lambda_{S \mid h}\left[\frac{1}{2} y_{h}^{*}\left(1-x_{h u}^{*}\right)-\left(1-y_{h}^{*}\right) x_{h h}^{*}\right]}{1-\frac{1}{2} f\left(y_{s}^{*}, y_{h}^{*}\right)}\right) .
\end{aligned}
$$


Because $\frac{\lambda_{S \mid s}}{1-\lambda_{S \mid s}}>\frac{\lambda_{S \mid h}}{1-\lambda_{S \mid h}}$ it must be that

$$
P_{h}\left(y_{s}^{*}, y_{h}^{*}\right) \leq 0 \text { implies that } P_{s}\left(y_{s}^{*}, y_{h}^{*}\right)<0 \text {. }
$$

From the optimality condition (9) in the text that, for convenience is rewritten here,

$$
P_{z}\left(y_{s}^{*}, y_{h}^{*}\right)=(>,<) 0 \text { as } y_{z}^{*} \in(0,1)\left(y_{z}^{*}=0 \text {, or } y_{z}^{*}=1\right), z \in\{s, h\},
$$

and from (B5) it follows that an equilibrium with $0 \leq y_{s}^{*}<1$ and $0<y_{h}^{*} \leq 1$ does not exist. Also, note that because

$$
P_{S}(0,0)=-\left(1-\lambda_{S \mid s}\right)\left(\frac{\lambda_{S \mid s}}{1-\lambda_{S \mid s}}(1-c)+\delta\left[g \lambda_{S \mid s} x_{s h}^{*}+(1-g) \lambda_{S \mid h} x_{h h}^{*}\right)<0,\right.
$$

an equilibrium with $\left(y_{s}^{*}, y_{h}^{*}\right)=(0,0)$ does not exist. Therefore, if equilibrium exists, then either $(1)\left(y_{s}^{*}, y_{h}^{*}\right)=(1,1)$, or $(2) y_{s}^{*}=1$ and $0<y_{h}^{*}<1$, or $(3)\left(y_{s}^{*}, y_{h}^{*}\right)=(1,0)$ or (4) $0<y_{s}^{*}<1$ and $y_{h}^{*}=0$.

Case 1 Suppose that in equilibrium $\left(y_{s}^{*}, y_{h}^{*}\right)=(1,1)$. Then the optimality conditions (9) become

$$
\begin{aligned}
P_{z} & (1,1)=\frac{1}{2} U_{z t}^{*}(1,1)+\frac{1}{2} U_{z u}^{*}-U_{z n}^{*}(1,1) \\
= & \frac{1}{2}\left[\lambda_{S \mid z} c+\left(1-\lambda_{S \mid z}\right) \delta R_{t}(1,1)\right]+\frac{1}{2} \min \left[\lambda_{S \mid z}, c\right]-\min \left[\lambda_{S \mid z}, c\right] \\
= & \frac{1}{2}\left(\lambda_{S \mid z} c+\left(1-\lambda_{S \mid z}\right) \delta R_{t}(1,1)-\min \left[\lambda_{S \mid z}, c\right]\right) \\
= & \frac{1}{2}\left(\left(1-\lambda_{S \mid z}\right) \delta\left(g \lambda_{S \mid s}\left(1-x_{s u}^{*}\right)+(1-g) \lambda_{S \mid h}\left(1-x_{h u}^{*}\right)\right)\right. \\
& \left.-\min \left[\lambda_{S \mid z}(1-c), c\left(1-\lambda_{S \mid z}\right)\right]\right) \\
= & \frac{1}{2}\left(\left(1-\lambda_{S \mid z}\right) \delta\left(g \lambda_{S \mid s} 1_{c>\lambda_{S \mid s}}+(1-g) \lambda_{S \mid h} 1_{c>\lambda_{S \mid h}}\right)\right. \\
& \left.-\min \left[\lambda_{S \mid z}(1-c), c\left(1-\lambda_{S \mid z}\right)\right]\right) \leq 0
\end{aligned}
$$

for $z \in\{s, h\}$.

For $c<\lambda_{S \mid h}$ the inequalities are satisfied since $P_{z}(1,1)=-\frac{1}{2} c\left(1-\lambda_{S \mid z}\right)<0$. For $\lambda_{S \mid h} \leq c<\lambda_{S \mid s}$ the inequalities become

$$
\begin{aligned}
& P_{S}(1,1)=\frac{1}{2}\left(\left(1-\lambda_{S \mid s}\right) \delta(1-g) \lambda_{S \mid h}-c\left(1-\lambda_{S \mid s}\right)\right) \leq 0, \\
& P_{h}(1,1)=\frac{1}{2}\left(\left(1-\lambda_{S \mid h}\right) \delta(1-g) \lambda_{S \mid h}-\lambda_{S \mid h}(1-c)\right) \leq 0 .
\end{aligned}
$$

Condition (B8) is satisfied by assumption. Condition (B9) is satisfied if

$$
c \leq 1-\left(1-\lambda_{S \mid h}\right) \delta(1-g)
$$


For $c \geq \lambda_{S \mid s}$ the inequalities (B7) become

$$
P_{z}(1,1)=\frac{1}{2}\left(\left(1-\lambda_{S \mid z}\right) \delta\left(g \lambda_{S \mid s}+(1-g) \lambda_{S \mid h}\right)-\lambda_{S \mid z}(1-c)\right) \leq 0 \text { for } z \in\{s, h\},
$$

or

$$
\begin{aligned}
c & \leq \min \left[1-\frac{1-\lambda_{S \mid h}}{\lambda_{S \mid h}} \delta\left(g \lambda_{S \mid s}+(1-g) \lambda_{S \mid h}\right), 1-\frac{1-\lambda_{S \mid s}}{\lambda_{S \mid s}} \delta\left(g \lambda_{S \mid s}+(1-g) \lambda_{S \mid h}\right)\right] \\
& =1-\frac{1-\lambda_{S \mid h}}{\lambda_{S \mid h}} \delta\left(g \lambda_{S \mid s}+(1-g) \lambda_{S \mid h}\right) .
\end{aligned}
$$

Therefore, in equilibrium $\left(y_{s}^{*}, y_{h}^{*}\right)=(1,1)$ if and only if

$$
c \leq c_{1}
$$

where

$$
c_{1} \equiv\left\{\begin{array}{l}
1-\left(1-\lambda_{S \mid h}\right) \delta(1-g), \text { if } \lambda_{S \mid s} \geq 1-\left(1-\lambda_{S \mid h}\right) \delta(1-g) \\
\lambda_{S \mid s}, \text { if } 1-\left(1-\lambda_{S \mid h}\right) \delta\left(\frac{g \lambda_{S \mid s}}{\lambda_{S \mid h}}+1-g\right) \leq \lambda_{S \mid s}<1-\left(1-\lambda_{S \mid h}\right) \delta(1-g) \\
1-\left(1-\lambda_{S \mid h}\right) \delta\left(\frac{g \lambda_{S \mid s}}{\lambda_{S \mid h}}+1-g\right), \text { if } \lambda_{S \mid s}<1-\left(1-\lambda_{S \mid h}\right) \delta\left(\frac{g \lambda_{S \mid s}}{\lambda_{S \mid h}}+1-g\right)
\end{array} .\right.
$$

Case 2 Suppose that in equilibrium $y_{s}^{*}=1$ and $0<y_{h}^{*}<1$. Then it must be that

$$
c>c_{1}
$$

because condition (B13) cannot be satisfied. Note that (B14) implies that in equilibrium

$$
c>\lambda_{S \mid h}+\left(1-\lambda_{S \mid h}\right) \delta(1-g)\left(1-y_{h}^{*}\right) \lambda_{S \mid h} .
$$

To see why suppose that condition $c \leq \lambda_{S \mid h}+\left(1-\lambda_{S \mid h}\right) \delta(1-g)\left(1-y_{h}^{*}\right) \lambda_{S \mid h}$ is satisfied. Then we have $U_{h h}^{*}\left(1, y_{h}^{*}\right)=c$, which implies that $y_{h}^{*}=1 \leq y_{s}^{*}=1$. This contradicts (B14).

Hence, the optimality condition for the rate of reporting suspicions by an asymptomatic agent in (9) becomes

$$
\begin{aligned}
& P_{h}\left(1, y_{h}^{*}\right)=\left(1-\frac{1}{2} f\left(1, y_{h}^{*}\right)\right) U_{h t}^{*}\left(1, y_{h}^{*}\right)+\frac{1}{2} f\left(1, y_{h}^{*}\right) U_{h u}^{*}-U_{h h}^{*}\left(1, y_{h}^{*}\right) \\
& =\left(\frac{1}{2} g+(1-g)\left(1-\frac{1}{2} y_{h}^{*}\right)\right)\left(\lambda_{S \mid h} c\right. \\
& \left.\quad+\left(1-\lambda_{S \mid h}\right) \delta \frac{\frac{1}{2} g \lambda_{S \mid s} 1_{c>\lambda_{S \mid s}}+(1-g)\left(1-\frac{1}{2} y_{h}^{*}\right) \lambda_{S \mid h}}{\frac{1}{2} g+(1-g)\left(1-\frac{1}{2} y_{h}^{*}\right)}\right)
\end{aligned}
$$




$$
\begin{aligned}
& +\frac{1}{2}\left(g+(1-g) y_{h}^{*}\right) \lambda_{S \mid h}-\left(\lambda_{S \mid h}+\left(1-\lambda_{S \mid h}\right) \delta(1-g)\left(1-y_{h}^{*}\right) \lambda_{S \mid h}\right) \\
= & \left(\frac{1}{2} g+(1-g)\left(1-\frac{1}{2} y_{h}^{*}\right)\right) \lambda_{S \mid h} c+\left(1-\lambda_{S \mid h}\right) \delta\left(\frac{1}{2} g \lambda_{S \mid s} 1_{c>\lambda_{S \mid s}}\right. \\
& \left.+(1-g)\left(1-\frac{1}{2} y_{h}^{*}\right) \lambda_{S \mid h}\right) \\
& +\frac{1}{2}\left(g+(1-g) y_{h}^{*}\right) \lambda_{S \mid h}-\left(\lambda_{S \mid h}+\left(1-\lambda_{S \mid h}\right) \delta(1-g)\left(1-y_{h}^{*}\right) \lambda_{S \mid h}\right)=0 .
\end{aligned}
$$

Solving for $y_{h}^{*}$ yields

$$
y_{h}^{*}=\frac{\left(1-\frac{1}{2} g\right) \lambda_{S \mid h}(1-c)-\frac{1}{2} \delta g\left(1-\lambda_{S \mid h}\right) \lambda_{S \mid s} 1_{c>\lambda_{S \mid s}}}{\frac{1}{2}\left(1-c+\left(1-\lambda_{S \mid h}\right) \delta\right)(1-g) \lambda_{S \mid h}} \in(0,1)
$$

if and only if

$$
c_{1}<c<c_{2}
$$

where

$$
c_{2} \equiv\left\{\begin{array}{ll}
1-\frac{\delta g \lambda_{S \mid s}\left(1-\lambda_{S \mid h}\right)}{(2-g) \lambda_{S \mid h}}, & \text { if } 1-\frac{\delta g \lambda_{S \mid s}\left(1-\lambda_{S \mid h}\right)}{(2-g) \lambda_{S \mid h}}>\lambda_{S \mid s} \\
\lambda_{S \mid s}, & \text { if } 1-\frac{\delta g \lambda_{S \mid s}\left(1-\lambda_{S \mid h}\right)}{(2-g) \lambda_{S \mid h}} \leq \lambda_{S \mid s}
\end{array} .\right.
$$

From (B5) and (B16), it follows that $P_{s}\left(1, y_{h}^{*}\right)<0$, which verifies that in equilibrium $y_{s}^{*}=1$ and $0<y_{h}^{*}<1$ if and only if (B18) is satisfied.

Case 3 Suppose that in equilibrium $\left(y_{s}^{*}, y_{h}^{*}\right)=(1,0)$. Then it must be that

$$
c \geq c_{2}
$$

The optimality condition (9) for an asymptomatic agent becomes

$$
\begin{aligned}
P_{h}(1,0)= & \left(1-\frac{1}{2} f(1,0)\right) U_{h t}^{*}(1,0)+\frac{1}{2} f(1,0) U_{h u}^{*}-U_{h h}^{*}(1,0) \\
= & \left(1-\frac{1}{2} g\right)\left(\lambda_{S \mid h} c+\left(1-\lambda_{S \mid h}\right) \delta \frac{\frac{1}{2} g \lambda_{S \mid s} 1_{c>\lambda_{S \mid s}}+(1-g) \lambda_{S \mid h}}{1-\frac{1}{2} g}\right) \\
& +\frac{1}{2} g \lambda_{S \mid h}-\left(\lambda_{S \mid h}+\left(1-\lambda_{S \mid h}\right) \delta(1-g) \lambda_{S \mid h}\right) \\
= & \left(1-\frac{1}{2} g\right) \lambda_{S \mid h} c+\left(1-\lambda_{S \mid h}\right) \delta\left(\frac{1}{2} g \lambda_{S \mid s} 1_{c>\lambda_{S \mid s}}+(1-g) \lambda_{S \mid h}\right) \\
& +\frac{1}{2} g \lambda_{S \mid h}-\left(\lambda_{S \mid h}+\left(1-\lambda_{S \mid h}\right) \delta(1-g) \lambda_{S \mid h}\right) \geq 0,
\end{aligned}
$$


which is implied by (B19).

If $c \leq \lambda_{S \mid s}+\left(1-\lambda_{S \mid s}\right) \delta(1-g) \lambda_{S \mid h}$, then from (B2) it follows that a symptomatic agent reports suspicions with probability one. For $c>\lambda_{S \mid s}+\left(1-\lambda_{S \mid s}\right) \delta(1-g) \lambda_{S \mid h}$, the optimality condition (9) for a symptomatic agent becomes

$$
\begin{aligned}
P_{S}(1,0)= & \left(1-\frac{1}{2} f(1,0)\right) U_{s t}^{*}(1,0)+\frac{1}{2} f(1,0) U_{s u}^{*}-U_{s h}^{*}(1,0) \\
= & \left(1-\frac{1}{2} g\right)\left(\lambda_{S \mid s} c+\left(1-\lambda_{S \mid s}\right) \delta \frac{\frac{1}{2} g \lambda_{S \mid s}+(1-g) \lambda_{S \mid h}}{1-\frac{1}{2} g}\right) \\
& +\frac{1}{2} g \lambda_{S \mid s}-\left(\lambda_{S \mid s}+\left(1-\lambda_{S \mid s}\right) \delta(1-g) \lambda_{S \mid h}\right) \\
= & -\left(1-\frac{1}{2} g\right) \lambda_{S \mid s}(1-c)+\frac{1}{2}\left(1-\lambda_{S \mid s}\right) \delta g \lambda_{S \mid s} \leq 0 .
\end{aligned}
$$

The last inequality can be equivalently rewritten as

$$
c \leq c_{3}
$$

where

$$
c_{3} \equiv 1-\delta g \frac{1-\lambda_{S \mid s}}{2-g} .
$$

Therefore, in equilibrium $\left(y_{s}^{*}, y_{h}^{*}\right)=(1,0)$ if and only if

$$
c_{2} \leq c \leq c_{3}
$$

Case 4 Suppose that in equilibrium $0<y_{s}^{*}<1$ and $y_{h}^{*}=0$. Then

$$
c>c_{3} \text {. }
$$

Furthermore, it must be that

$$
c>\lambda_{S \mid s}+\left(1-\lambda_{S \mid s}\right) \delta\left(g\left(1-y_{s}^{*}\right) \lambda_{S \mid s}+(1-g) \lambda_{S \mid h}\right) .
$$

To see why suppose that condition $c \leq \lambda_{S \mid s}+\left(1-\lambda_{S \mid s}\right) \delta\left(g\left(1-y_{s}^{*}\right) \lambda_{S \mid s}+(1-g) \lambda_{S \mid h}\right)$ is satisfied. Then we have $U_{s h}^{*}\left(y_{s}^{*}, 0\right)=c$, which implies that $y_{s}^{*}=1$. This contradicts (B26) because, as shown in the analysis of Cases 1-3, $y_{s}^{*}=1$ implies that $c \leq c_{3}$.

Using (B27) the optimality condition (9) for a symptomatic agent becomes

$$
\begin{aligned}
P_{s}\left(y_{s}^{*}, 0\right) & =\left(1-\frac{1}{2} f\left(y_{s}^{*}, 0\right)\right) U_{s t}^{*}\left(y_{s}^{*}, 0\right)+\frac{1}{2} f\left(y_{s}^{*}, 0\right) U_{s u}^{*}-U_{s h}^{*}\left(y_{s}^{*}, 0\right) \\
& =\left(1-\frac{1}{2} g y_{s}^{*}\right)\left(\lambda_{S \mid s} c+\left(1-\lambda_{S \mid s}\right) \delta \frac{g\left(1-\frac{1}{2} y_{s}^{*}\right) \lambda_{S \mid s}+(1-g) \lambda_{S \mid h}}{1-\frac{1}{2} g y_{s}^{*}}\right)
\end{aligned}
$$




$$
\begin{aligned}
& +\frac{1}{2} g y_{s}^{*} \lambda_{S \mid h}-\left(\lambda_{S \mid s}+\left(1-\lambda_{S \mid s}\right) \delta\left(g\left(1-y_{s}^{*}\right) \lambda_{S \mid s}+(1-g) \lambda_{S \mid h}\right)\right) \\
= & \frac{1}{2}\left(1-\lambda_{S \mid s}\right) \delta g y_{s}^{*} \lambda_{S \mid s}-\left(1-\frac{1}{2} g y_{s}^{*}\right) \lambda_{S \mid s}(1-c)=0 .
\end{aligned}
$$

Solving for $y_{s}^{*}$ yields

$$
y_{s}^{*}=2 \frac{1-c}{g\left(1-c+\delta\left(1-\lambda_{S \mid s}\right)\right)} \in(0,1) \text {, }
$$

where $y_{s}^{*}<1$ because, by assumption, $c>c_{3}$.

An asymptomatic agent prefers not to report suspicions because, from (B5) and (B28), it follows that

$$
P_{h}\left(y_{s}^{*}, 0\right)>0 .
$$

Summarizing these four cases, the unique equilibrium conditional rates of reporting suspicions (as part of the unique equilibrium of the entire game) are given by

$$
\left(y_{s}^{*}, y_{h}^{*}\right)=\left\{\begin{array}{l}
(1,1), \quad \text { if } c \leq c_{1} \\
\left(1, \hat{y}_{h}(c)\right), \quad \text { if } c_{1}<c<c_{2} \\
(1,0), \quad \text { if } c_{2} \leq c \leq c_{3} \\
\left(\hat{y}_{s}(c), 0\right), \quad \text { if } c>c_{3}
\end{array}\right.
$$

where

$$
\begin{aligned}
& \hat{y}_{h}(c) \equiv \frac{\lambda_{S \mid h}(1-c)\left(1-\frac{1}{2} g\right)-\frac{1}{2} \delta\left(1-\lambda_{S \mid h}\right) \lambda_{S \mid s} g 1_{c>\lambda_{S \mid s}}}{\frac{1}{2} \lambda_{S \mid h}(1-g)\left(\delta\left(1-\lambda_{S \mid h}\right)+1-c\right)} \in(0,1) \text { for } c_{1}<c<c_{2}, \\
& \hat{y}_{S}(c) \equiv \frac{2(1-c)}{g\left(\delta\left(1-\lambda_{S \mid s}\right)+1-c\right)} \in(0,1) \text { for } c>c_{3} .
\end{aligned}
$$

To derive the comparative statics results about $\delta, \alpha, \beta$, it will be convenient to rewrite the threshold values of the cost of disease control as

$$
\begin{aligned}
& c_{1}=\max \left[\min \left[1-\delta(1-\alpha) \beta, \frac{\alpha \beta}{\alpha \beta+(1-\alpha)(1-\beta)}\right], 1-\delta \frac{(1-\alpha) \beta}{1-\beta}\right] \\
& c_{2}=\max \left[1-\frac{\delta(1-\alpha) \beta^{2}}{(2-\alpha \beta-(1-\alpha)(1-\beta))(1-\beta)}, \frac{\alpha \beta}{\alpha \beta+(1-\alpha)(1-\beta)}\right], \text { and } \\
& c_{3}=1-\frac{\delta(1-\alpha)(1-\beta)}{2-\alpha \beta-(1-\alpha)(1-\beta)},
\end{aligned}
$$

and the equilibrium probabilities of reporting suspicions as 


$$
\begin{aligned}
& y_{h}^{*}=\min \left[\frac{\max \left[(1-\beta)(1-c)(2-\alpha \beta-(1-\alpha)(1-\beta))-\delta(1-\alpha) \beta^{2} 1_{c>\lambda_{S \mid s}}, 0\right]}{(1-\beta)(\delta(1-\alpha) \beta+(1-c)(\alpha(1-\beta)+(1-\alpha) \beta))}, 1\right], \\
& y_{s}^{*} \equiv \min \left[\frac{2(1-c)}{\delta(1-\alpha)(1-\beta)+(1-c)(\alpha \beta+(1-\alpha)(1-\beta))}, 1\right] .
\end{aligned}
$$

It can be verified that $y_{h}^{*}$ and $y_{s}^{*}$ are non-increasing in $\delta$ (and are strictly decreasing for $y_{h}^{*} \in(0,1)$ and $y_{s}^{*} \in(0,1)$, respectively).

Next we show that $y_{h}^{*}$ is non-decreasing in $\alpha$. Note that $c_{1}$ and $c_{2}$ increase with $\alpha$. For $c_{1}<c<\lambda_{S \mid s}$, differentiation yields

$$
\frac{\partial y_{h}^{*}}{\partial \alpha}=(1-c) \frac{(2-\beta) \delta \beta+(2 \beta-1)(1-c)}{(\delta(1-\alpha) \beta+(1-c)(\alpha(1-\beta)+(1-\alpha) \beta))^{2}}>0
$$

For $\lambda_{S \mid s}<c<c_{2}$, differentiation yields

$$
\frac{\partial y_{h}^{*}}{\partial \alpha}=(1-c) \frac{(2-\beta) \delta \beta-\left(1-2 \beta-\delta \beta^{2}\right)(1-c)}{(\delta(1-\alpha) \beta+(1-c)(\alpha(1-\beta)+(1-\alpha) \beta))^{2}}>0 .
$$

Now we show that $y_{s}^{*}$ is non-decreasing in $\alpha$. Note that $c_{3}$ increases with $\alpha$, and that differentiation of $y_{s}^{*}$ for $c>c_{3}$ yields

$$
\frac{\partial y_{s}^{*}}{\partial \alpha}=-\frac{2(1-c)(-\delta(1-\beta)+(1-c)(2 \beta-1))}{(\delta(1-\alpha)(1-\beta)+(1-c)(\alpha \beta+(1-\alpha)(1-\beta)))^{2}}>0,
$$

whenever $c>1-\frac{\delta(1-\beta)}{2 \beta-1}$. Because, by assumption,

$$
1-\frac{\delta(1-\beta)}{2 \beta-1}<c_{3}<c
$$

it follows that $y_{s}^{*}$ is non-decreasing in $\alpha$.

Finally, we show that $y_{s}^{*}$ increases with $\beta$. Note that $c_{3}$ increases with $\beta$ and that differentiation of $y_{s}^{*}$ for $c>c_{3}$ yields

$$
\frac{\partial y_{s}^{*}}{\partial \beta}=\frac{2(1-c)(\delta(1-\alpha)+(1-c)(1-2 \alpha))}{(\delta(1-\alpha)(1-\beta)+(1-c)(\alpha \beta+(1-\alpha)(1-\beta)))^{2}}>0 .
$$

For $1-2 \alpha<0$ the inequality in (B32) holds if $c>1-\frac{\delta(1-\alpha)}{2 \alpha-1}$, which is true because, by assumption,

$$
1-\frac{\delta(1-\alpha)}{2 \alpha-1}<c_{3}<c
$$

For $1-2 \alpha \geq 0$ the inequality in (B32) holds because all terms are positive. Hence, it follows that $y_{s}^{*}$ increases with $\beta$. 


\section{Appendix C}

Proof of Corollary 1 Taking the limits as $\alpha \rightarrow 0$ yields

$$
\begin{aligned}
\lim _{\alpha \rightarrow 0} c_{2} & =\max \left[1-\frac{\delta g \lambda_{S \mid s}\left(1-\lambda_{S \mid h}\right)}{(2-g) \lambda_{S \mid h}}, \lambda_{S \mid s}\right] \\
& =\max \left[1-\frac{\delta(\beta(\alpha))^{2}}{1-(\beta(\alpha))^{2}}, \frac{\beta(\alpha)}{\beta(\alpha)+(1-\beta(\alpha)) / \alpha}\right]=0,
\end{aligned}
$$

and

$$
\lim _{\alpha \rightarrow 0} c_{3}=1-\delta \frac{g\left(1-\lambda_{S \mid s}\right)}{2-g}=\lim _{\alpha \rightarrow 0} 1-\delta \frac{1-\beta(\alpha)}{1+\beta(\alpha)}=1 .
$$

The result follows from Proposition 1 by continuity.

Proof of Lemma 2 If $c \geq \lambda_{S \mid s}+\delta\left(1-\lambda_{S \mid s}\right) \lambda_{S \mid s}$, by Lemma 1, in equilibrium an untested agent of any type does control the disease. Therefore, for given rates of reporting suspicions, the expected welfare is given by

$$
\begin{aligned}
W\left(y_{s}, y_{h}\right)= & g^{2}\left(\left(1-\left(1-y_{s}\right)^{2}\right)\left(\lambda_{S \mid s} c+\delta\left(1-\lambda_{S \mid s}\right) \lambda_{S \mid s}+\lambda_{S \mid s}\right)\right. \\
& \left.+2\left(1-y_{s}\right)^{2}\left(\lambda_{S \mid s}+\delta\left(1-\lambda_{S \mid s}\right) \lambda_{S \mid s}\right)\right) \\
& +2(1-g) g\left(y_{s}\left(1-\frac{1}{2} y_{h}\right)\left(\lambda_{S \mid s} c+\delta\left(1-\lambda_{S \mid s}\right) \lambda_{S \mid h}+\lambda_{S \mid h}\right)\right. \\
& \left.+\left(1-\frac{1}{2} y_{s}\right) y_{h}\left(\lambda_{S \mid h} c+\delta\left(1-\lambda_{S \mid h}\right) \lambda_{S \mid s}+\lambda_{S \mid s}\right)\right) \\
& \left.+\left(1-y_{s}\right)\left(1-y_{h}\right)\left(\lambda_{S \mid s}+\delta\left(1-\lambda_{S \mid s}\right) \lambda_{S \mid h}+\lambda_{S \mid h}+\delta\left(1-\lambda_{S \mid h}\right) \lambda_{S \mid s}\right)\right) \\
& +(1-g)^{2}\left(\left(1-\left(1-y_{h}\right)^{2}\right)\left(\lambda_{S \mid h} c+\delta\left(1-\lambda_{S \mid h}\right) \lambda_{S \mid h}+\lambda_{S \mid h}\right)\right. \\
& \left.+2\left(1-y_{h}\right)^{2}\left(\lambda_{S \mid h}+\delta\left(1-\lambda_{S \mid h}\right) \lambda_{S \mid h}\right)\right) .
\end{aligned}
$$

Differentiation with respect to $y_{s}$ yields

$$
\begin{aligned}
\frac{\partial W\left(y_{s}, y_{h}\right)}{\partial y_{s}}= & g^{2}(-2)\left(1-y_{s}\right)\left(2\left(\lambda_{S \mid s}+\delta\left(1-\lambda_{S \mid s}\right) \lambda_{S \mid s}\right)\right. \\
& \left.-\lambda_{S \mid s} c-\delta\left(1-\lambda_{S \mid s}\right) \lambda_{S \mid s}-\lambda_{S \mid s}\right) \\
& +2(1-g) g\left(\left(1-\frac{1}{2} y_{h}\right)\left(\lambda_{S \mid s} c+\delta\left(1-\lambda_{S \mid s}\right) \lambda_{S \mid h}+\lambda_{S \mid h}\right)\right. \\
& \left.-\frac{1}{2} y_{h}\left(\lambda_{S \mid h} c+\delta\left(1-\lambda_{S \mid h}\right) \lambda_{S \mid s}+\lambda_{S \mid s}\right)\right) \\
& \left.-\left(1-y_{h}\right)\left(\lambda_{S \mid s}+\delta\left(1-\lambda_{S \mid s}\right) \lambda_{S \mid h}+\lambda_{S \mid h}+\delta\left(1-\lambda_{S \mid h}\right) \lambda_{S \mid s}\right)\right) \\
\leq & -2(1-g) g \lambda_{S \mid s}\left(1-c+\delta\left(1-\lambda_{S \mid h}\right)\right)<0 \text { for all } y_{s}, y_{h} .
\end{aligned}
$$


Therefore, at optimum $y_{s}=1$. Differentiation with respect to $y_{h}$ at $y_{s}=1$ yields

$$
\begin{aligned}
& \frac{\partial W\left(1, y_{h}\right)}{\partial y_{h}}=(1-g) g\left(\lambda_{S \mid h} c+\delta\left(1-\lambda_{S \mid h}\right) \lambda_{S \mid s}+\lambda_{S \mid s}-\lambda_{S \mid s} c\right. \\
& \left.\quad-\delta\left(1-\lambda_{S \mid s}\right) \lambda_{S \mid h}-\lambda_{S \mid h}\right)-2(1-g)^{2}\left(1-y_{h}\right)\left(2\left(\lambda_{S \mid h}+\delta\left(1-\lambda_{S \mid h}\right) \lambda_{S \mid h}\right)\right. \\
& \left.\quad-\lambda_{S \mid h} c-\delta\left(1-\lambda_{S \mid h}\right) \lambda_{S \mid h}-\lambda_{S \mid h}\right) \\
& \quad \geq(1-g) g\left(\lambda_{S \mid s}-\lambda_{S \mid h}\right)(1-c+\delta)-2(1-g)^{2} \lambda_{S \mid h}\left(1-c+\delta\left(1-\lambda_{S \mid h}\right)\right)>0,
\end{aligned}
$$

where the last inequality follows because, by assumption, $g \lambda_{S \mid s}>(2-g) \lambda_{S \mid h}$. This establishes that $\left(y_{s}, y_{h}\right)=(1,0) \in \arg \min _{y_{s}, y_{h}} W\left(y_{s}, y_{h}\right)$.

\section{Appendix D}

The equilibrium outcome does not change in the presence of uncertainty about $\delta$. Suppose that $\delta$ is drawn independently from all other elements of the model from some distribution $F$ with support on $[0,1]$. Then the expected conditional probability of disease transmission (with respect to $\delta$ ) is given by

$$
\begin{aligned}
& E_{\delta}\left[\operatorname{Pr}\left(\theta_{i, 1}=1 \mid \theta_{A, 0}, \theta_{B, 0}, e_{A}, e_{B}\right) \mid \theta_{A, 0}, \theta_{B, 0}, e_{A}, e_{B}\right] \\
& \quad=E_{\delta}\left[\operatorname{Pr}\left(\theta_{i, 1}=1 \mid \theta_{A, 0}, \theta_{B, 0}, e_{A}, e_{B}\right)\right] \\
& \quad=\left\{\theta_{i, 0}+E[\delta]\left(1-\theta_{i, 0}\right)\left(1-e_{-i}\right) \theta_{-i, 0}\right\}\left(1-e_{i}\right) .
\end{aligned}
$$

The first equality follows because $\delta$ is drawn independently from the initial disease states. The second equality follows because the conditional probability of disease transmission is linear in $\delta$. Since the agents minimize their expected costs, allowing for uncertainty about $\delta$ leads to an equivalent analysis where $\delta$ is replaced by its expected value $E[\delta]$.

\section{References}

Adhvaryu, A.: Learning, misallocation, and technology adoption: evidence from new malaria therapy in Tanzania. Rev. Econ. Stud. 81, 1331-1365 (2015)

Ambrus, A., Takahashi, S.: Multi-sender cheap talk with restrictive state space. Theor. Econ. 3, 1-27 (2008) Anderson, R., May, R.: Population biology of infectious diseases. Part I. Nature 280, 361-367 (1979)

Austen-Smith, D.: Interested experts and policy advice: multiple referrals under open rule. Games Econ. Behav. 5, 1-43 (1993)

Battaglini, M.: Multiple referrals and multidimensional cheap talk. Econometrica 70, 1379-1401 (2002)

Bauch, C.T., Galvani, A.P., Earn, D.J.D.: Group interest versus self-interest in smallpox vaccination policy. Proc. Natl. Acad. Sci. 100, 10564-10567 (2003)

Boozer, M.A., Philipson, T.J.: The impact of public testing for human immunodeficiency virus. J. Hum. Resour. 35, 419-446 (2000)

Brown, M., Trautmann, S., Vlahu, R.: Understanding bank-run contagion. Manag. Sci. (2016) (forthcoming)

Cohen, J., Dupas, P., Schaner, S.: Price subsidies, diagnostic tests and targeting of malaria treatment: evidence from a randomized controlled trial. Am. Econ. Rev. 105, 609-645 (2015)

Crawford, V., Sobel, J.: Strategic information transmission. Econometrica 50, 1431-1451 (1982)

Daley, D.J., Gani, J.: Epidemic Modelling: An introduction. Cambridge University Press, Cambridge (1999)

Epstein, D.: Partisan and bipartisan signaling in congress. J. Law Econ. Organ. 14, 183-204 (1998) 
Fenichel, E.P., Castillo-Chavez, C., Ceddia, M.G., Chowell, G., Gonzalez Parra, P.A., Hickling, G.J., Holloway, G., Horan, R., Morin, B., Perrings, C., Springborn, M., Velazquez, L., Villalobos, C.: Adaptive human behavior in epidemiological models. Proc. Natl. Acad. Sci. 108, 6306-6311 (2011)

Funk, S., Salathé, M., Jansen, V.A.A.: Modelling the influence of human behavior on the spread of infectious diseases: a review. J. R. Soc. Interface 7, 1247-1256 (2010)

Funk, S., Bansal, S., Bauch, C.T., Eames, K.T.D., Edmunds, W.J., Galvani, A.P., Klepac, P.: Nine challenges in incorporating the dynamics of behavior in infectious diseases models. Epidemics 10, 21-25 (2015)

Gates, G.: The next epidemic_-lessons from Ebola. New Engl. J. Med. 372, 1381-1384 (2015)

Gilligan, T., Krehbiel, K.: Asymmetric information and legislative rules with a heterogeneous committee. Am. J. Polit. Sci. 33, 459-490 (1989)

Godlonton, S., Thornton, R.L.: Peer effects in learning HIV results. J. Dev. Econ. 97, 118-129 (2012)

Godlonton, S., Thornton, R.L.: Learning from others' HIV testing: updating beliefs and responding to risk. Amer. Econ. Rev. P\&P 103, 439-444 (2013)

Goenka, A., Liu, L.: Infectious diseases and endogenous fluctuations. Econ. Theory 50, 125-149 (2012)

Goenka, A., Liu, L., Nguyen, M.H.: Infectious diseases and economic growth. J. Math. Econ. 50, 34-53 (2014)

Gong, E.: HIV testing and risky sexual behavior. Econ. J. 125, 32-60 (2014)

Klepac, P., Laxminarayan, R., Grenfell, B.: Synthesizing epidemiological and economic optima for control of immunizing infections. Proc. Natl. Acad. Sci. 108, 14366-14370 (2011)

Klepac, P., Bjørnstad, O., Metcalf, C., Grenfell, B.: Optimizing reactive responses to outbreaks of immunizing infections: balancing case management and vaccination. PLoS ONE 7, e41428 (2012)

Klepac, P., Megiddo, I., Grenfell, B., Laxminarayan, R.: Self-enforcing regional vaccination agreements. J. R. Soc. Interface 13, 20150907 (2016)

Kremer, M.: Integrating behavioral choice into epidemiological models of AIDS. Q. J. Econ. 111, 549-573 (1996)

Krishna, V., Morgan, J.: A model of expertise. Q. J. Econ. 116, 747-775 (2001)

Lessells, R., Cooke, G., Newell, M., Godfrey-Faussett, P.: Evaluation of tuberculosis diagnostics: establishing an evidence base around the public health impact. J. Infect. Dis. 204, 1187-1195 (2011)

Malani, A., Laxminarayan, R.: Incentives for reporting infectious disease outbreaks. J. Hum. Res. 46, 176-202 (2011)

McGee, A., Yang, H.: Cheap talk with two senders and complementary information. Games Econ Behav 79, 181-191 (2013)

Mesnard, A., Seabright, P.: Escaping epidemics through migration? Quarantine measures under incomplete information about infection risk. J. Pub. Econ. 93, 931-938 (2009)

Metzler, M.I., Mills McNeill, K., Miller, J.D.: Laboratory surge capacity and Pandemic Influenza. Emerg. Infect. Dis. 16, 147-148 (2010)

Morgan, J., Stocken, P.: Information aggregation in polls. Am. Econ. Rev. 98, 864-896 (2008)

Nguyen, C., Carlson, J.: Optimizing real-time vaccine allocation in a stochastic SIR model. PLoS ONE 11, e0152950 (2016)

Olmstead, A., Rhode, P.: An impossible undertaking: the eradication of bovine tuberculosis in the United States. J. Econ. Hist. 64, 734-772 (2004)

Perrings, C., Castillo-Chavez, C., Chowell, G., Daszak, P., Fenichel, E.P., Finnoff, D., Horan, R.D., Kilpatrick, A.M., Kinzig, A.P., Kuminoff, N.V., Levin, S., Morin, B., Smith, K.F., Springborn, M.: Merging economics and epidemiology to improve the prediction and management of infectious disease. EcoHealth 11, 464-475 (2014)

Philipson, T., Posner, R.A.: A theoretical and empirical investigation of the effects of public health subsidies for STD testing. Q. J. Econ. 110, 445-474 (1995)

Schäferhoff, M., Fewer, S., Kraus, J., Richter, E., Summers, L.H., Sundewall, J., Yamey, G., Jamison, D.T.: How much donor financing for health is channeled to global versus country-specific aid functions? Lancet 386, 12-18 (2015)

Shavell, S.: Acquisition and disclosure of information prior to sale. RAND J. Econ. 25, 20-36 (1994)

The TDR Diagnostics Evaluation Expert Panel (DEEP): Evaluation of diagnostic tests for infectious diseases: general principles. Nat. Rev. Microbiol. 6, S16-S28 (2008)

Thornton, R.L.: The demand for, and impact of, learning HIV status. Am. Econ. Rev. 98, 1829-1863 (2008)

Ward, C.J.: Influenza vaccination campaigns: is an ounce of prevention worth a pound of cure? Am. Econ. J. Appl. Econ. 6, 38-72 (2014)

Wolinsky, A.: Eliciting information from multiple experts. Games Econ. Behav. 41, 141-160 (2002) 